\title{
Influential Article Review - An Identity Approach to Sex Variations in Entrepreneurs' Industry Growth Plans
}

\author{
Firat Cassidy
}

Alena Suarez

Isis Fountain

This paper examines business growth and entrepreneurship. We present insights from a highly influential paper. Here are the highlights from this paper: The present empirical study explores the effects of gender role and cultural identity (masculinity and femininity) in mens' and womens' business growth intentions in established firms. A questionnaire survey was completed by 572 business owners (286 females). Results from moderated mediation regression analyses found that masculinity and femininity fully mediated the effects of entrepreneurs' sex on business growth intentions. Females who had higher femininity orientation and independent self-construal reported lower growth intention compared to those with lower independent self-construal. The study extends theoretical and empirical research on the effects of identity on business growth intentions while applications of the results are discussed. For our overseas readers, we then present the insights from this paper in Spanish, French, Portuguese, and German.

Keywords: entrepreneurs, growth intentions, identity, gender, self-construal

\section{SUMMARY}

- The present study had two primary goals: to examine whether and how gender role identity can explain effects of entrepreneurs' sex on business growth intentions in established firms and to explore the role of independent self-construal regarding this possible mediation effect. In particular, we were interested in finding out whether independent self-construal moderates the indirect effects of sex on business growth intentions. Results from conditional process analysis suggested that masculinity and femininity fully mediated the effects of entrepreneurs' biological sex on his/her business growth intentions. Moreover, results provided evidence that the indirect effect of sex on business growth intentions was contingent on entrepreneurs' independent self-construal.

- The primary contribution of this research is that it is the first to empirically demonstrate that gender identity constitutes an important part in conceptual models that explain sex differences in business growth intentions. Further research is needed to explore the reasons behind females' lower growth intention. Certainly, issues of individual motivation factors such as ego-depletion could be explored. However, issues to do with the social context should also be explored. This research took place in country at southern Europe, where the general cultural mandate is towards interdependence. Self-construal orientations have shown to interact with nation-level cultural 
orientation to influence entrepreneurship intentions, and further research should explore the contribution of those in women entrepreneurship.

- The findings of the present study demonstrate that masculinity and femininity completely mediated the influence of sex on growth intentions, after controlling for the effects of perceived behavioral control and internal locus of control. The precise measure of femininity as well as masculinity allows us to look at how gender identity affects the sex and growth intentions relationships. Moreover, educators should gain a better general understanding of how womens' entrepreneurial intentions are formed, as well as a specific understanding of how entrepreneur' gender identity and independent self-construal merge into the intent to growth a business.

- Our study has also some implications for innovation policy. Contemporary research suggests that public resource distribution to innovation processes have to large extent directed to manufacturing industries and new technologies, both primarily employing men as employees and entrepreneurs and not to the service industries employing mostly women or both men and women to the same extent. This suggests that innovation policies have primarily promoted innovation in a narrow spectrum of sectors and innovation types.

- Although in the entrepreneurship the individual is visible, in the innovation policy, the individual is made invisible, yet research suggests that we can still see a clear construction of masculinity in how and where innovation is expected to come about. Moreover, men are ascribed a normative role in innovation policies and innovation networks.

\section{HIGHLY INFLUENTIAL ARTICLE}

We used the following article as a basis of our evaluation:

Zampetakis, L.A., Bakatsaki, M., Kafetsios, K., \& Moustakis, V.S. (2016). Sex differences in entrepreneurs' business growth intentions: An identity approach. Journal of Innovation and Entrepreneurship, 5(1).

This is the link to the publisher's website: https://innovation-entrepreneurship.springeropen.com/articles/10.1186/s13731-016-0057-5

\section{INTRODUCTION}

The development and growth of existing business is a process with many societal benefits including job and wealth creation and the advancement of innovation (Tang \& Koveos, 2004; Van Praag \& Versloot, 2007). Entrepreneurs' decision for growing their business is complex, is neither linear nor dependent on a limited number of factors (Miller et al. 2013). Entrepreneurs not only have to make important decisions for different tasks which are different in nature but also have to ensure that those decisions are the right ones not only for their business but also for themselves. While some entrepreneurs are pleased to be selfemployed in a small scale, others have growth aspirations for their ventures (Shane, 2009).

Research clearly delineates that women-owned businesses tend to have lower levels of growth and remain smaller than men-owned businesses (Cliff, 1998; Coleman, 2016; Davis \& Shaver, 2012), suggesting the existence of a gap between men and women in entrepreneurship; this is an issue that is attracting increasing academic attention (Coleman, 2016). However, much of that research has investigated differences in the levels of start-up activity between men and women (see Jennings \& Brush, 2013). Little research has examined the growth aspirations of ventures led by women. Compared to male entrepreneurs, female entrepreneurs tend to pursue noneconomic goals such as balancing work and family roles and have preferences for employee relationship and society satisfiers which in turn may detract from economic performance or growth (Eddleston \& Powell, 2008; Jennings \& Brush, 2013).

As noted by Henry et al. (2016), normative representations of entrepreneurship are dominated by masculinity. This marginalizes female entrepreneurs and renders them invisible. The authors note that 
whilst the awareness that gender (masculine and feminine) is socially constructed and corresponds to a learned set of behaviors that does not refer to simply the biological sex (male/man and female/woman) of the entrepreneur seems widespread among scholars, several detrimental themes related to women are persistent. For instance, many scholars use male norms to judge women's activities merely comparing men and women, with little or no attention paid to constructions of gender (Ahl, 2006; Lewis, 2006). However, this practice neglects the fact that masculine and feminine aspects will be incorporated into the process of business growth for both men and women. That is to say, gender could be a vital aspect of business growth intention.

Taken together, to date, research does not provide conclusive explanations for the variation in the growth trajectory that men and women would like their venture to follow (i.e., their growth intention) (Bulanova et al. 2016; Dutta \& Thornhill, 2008). Growth intention is considered an essential characteristic of entrepreneurial behavior and a key element in understanding venture development and growth (SadlerSmith et al. 2003). Moreover, intentions have proven to be the best predictor of planned behavior (Ajzen, 1991), especially if the phenomenon involves unpredictable time lags, planning, and a high degree of cognitive processing, such as business growth (Krueger et al. 2000). Growth intentions are considered a key predictor of actual business growth (Delmar \& Wiklund, 2008; Wiklund \& Shepherd, 2003).

Considerable evidence suggests that an individual's identity (or concept of self) in general (Fauchart \& Gruber, 2011; Hoang \& Gimeno, 2010) and gender identity (or related gender roles) in particular (Eddleston \& Powell, 2008; Gupta et al., 2009) can contribute to substantial differences between men and women in entrepreneurial behavior. People tend to differ in the extent to which they incorporate gender roles into their self-concepts.

In the present study, we propose that entrepreneurs' gender identity or the extent to which entrepreneurs possess traits associated with traditional gender stereotypes (Bem's Sex Role Inventory, 1981; Mueller, 2004; Wood \& Eagly, 2010) is an important cognitive mechanism that relates entrepreneurs' sex to business growth intentions. We argue that gender identity shapes the way entrepreneurs view themselves, how they understand the world around them and approach other people, but also what they aim to achieve in the future (Eddleston \& Powell, 2008; Hoang \& Gimeno, 2010).

However, the mechanisms by which sex and gender identity exert influence on entrepreneurs' growth intentions and the moderating influences that constitute boundary conditions of the theory are in need of further investigation. Self-construal is such a potential moderator of the relationship between gender, gender identity, and growth intentions. Self-construal refers to individuals' culturally contingent thoughts, feelings, and actions that are concerned with one's understanding of the self as connected to others (interdependent self-construal) or distinct from others (independent self-construal) (Markus \& Kitayama, 1991). Individuals' exposure to certain conditions can increase the likelihood they exhibit independent (individualistic) or interdependent (collectivistic) tendencies (Hong et al., 2000). The concepts of "entrepreneur" and "entrepreneurship" are considered male-gendered (Ahl, 2006; Lewis, 2006). Cues or symbols in the environment that entrepreneurs utilize to operate their business could make independent self-construal more accessible (Hong et al., 2000), since the masculine identity highlights individuals' unique attributes (Cross \& Madson, 1997). A systematic consideration of the potential moderating effects of self-construal will contribute to a fuller understanding of the conditions under which the effects of sex on growth intentions are more likely to hold.

In sum, the aim of this article is to deepen the understanding of the factors that influence business growth motivation in established firms. Towards this end, one major aim of the present study is to examine the relationship between entrepreneurs' sex, gender identity, and business owner's growth intentions of their established ventures. We reasoned that an exploration into the growth intentions of business owners of established firms might help explain sex differences in the decision to grow. A second major purpose of the present study is to focus on entrepreneurs' self-construal as a potential moderator of the relationship between sex and gender identity and between gender identity and business growth intention (see conceptual model in Fig. 1). To the best of our knowledge, to date, there has been little research explaining the influence of these domains and entrepreneurs' intention for the growth established business. However, this is 
important considering that individuals start and operate their ventures for a variety of reasons other than growth or maximizing economic returns (Wiklund et al. 2003).

The paper commences with an analysis of business growth intentions; this is followed by an evaluation of the influence of gender identity on growth intentions and the moderating role of self-construal. Next, we describe our sample, research methods, and data analysis techniques which include ANOVA and moderated mediation analyses. Finally, we discuss the outcomes of the empirical analysis and the limitations of the research, present the practical implication of research results, and propose areas for further research.

\section{Growth Intention and Entrepreneurs' Gender Identity}

Business growth is a multidimensional and complex phenomenon that involves careful planning and thinking on the part of the entrepreneur. As such, business growth can be considered a deliberate and planned intentional behavior and consequently applicable for intention models (Ajzen, 1991; Krueger et al., 2000). Growth intentions are central to the understanding of business growth as they are the first step in the process and involve a purposive element with specific steps to reach the goal (Delmar \& Wiklund, 2008; Venugopal, 2016). Intentions have been identified as a key predictor of actual behavior, across a wide range of different behaviors including business startup (Delmar \& Wiklund, 2008; Wiklund \& Shepherd, 2003). Moreover, research provides evidence that the proportion of entrepreneurs with growth intentions in the population is a significant predictor of economic growth compared to self-employment rates or general start-up rates (Stam et al., 2009).

Up to date, research on entrepreneurs' growth intentions has produced mixed results regarding empirical differences between men and women and not all scholars agree that women and men differ in their overall business growth intent (Cassar, 2006; Davis \& Shaver, 2012). For instance, Cassar (2006) found that women entrepreneurs had lower estimates of future revenues than did men. However, Menzies et al. (2004), in a study conducted in Canada, found no statistically significant differences between women and men in their expressed preferences for unrestrained growth. In the present study, we propose that gender socialization processes may explain differences between male and female entrepreneurs' business growth intentions.

Contemporary research in entrepreneurship suggests that decisions such as the growth of established ventures are intimately intertwined with the entrepreneurs' identity (Shepherd \& Haynie 2009). Identity is a fluid social process that has a range of conceptual meanings and theoretical roles associated with it (Fauchart \& Gruber, 2011; Hoang \& Gimeno, 2010). It can be viewed as peoples' representation of the internalization and incorporation of socially held behavioral expectations. Moreover, identity can be regarded as a fundamental bridging concept between the individual and the social (Hoang \& Gimeno, 2010). Individuals are socially constructed through social interactions and that they acquire throughout their lives diverse and multiple social identities. In the case of the entrepreneurial process, Ireland and Webb (2007) note in their review that for entrepreneurs, the entrepreneurial process is based on and driven by selfidentities. Recent conceptual models of the role of identity in entrepreneurship propose strong links between entrepreneurs' self-concept and entrepreneurial actions and outcomes (Shepherd \& Haynie 2009), but to date, empirical research is limited (Farmer et al. 2011). Gender identity is considered an essential element of a many-sided conception of the self that situates individuals within social structures (Eddleston \& Powell, 2008; Wood \& Eagly, 2010). Gender is different from sex (Ahl, 2006). Researchers have distinguished sex from gender during the 1970s and 1980s to distinguish between biological characteristic of males and females and the meanings that societies and individuals ascribe to male and female categories (Bem's Sex Role Inventory, 1981; Wood \& Eagly, 2010). Individuals throughout the life course are taught (implicitly or explicitly) which behaviors and roles are desirable for men or women in society. As such, most individuals accept or internalize the cultural meanings associated with their sex meanings. Gender identity represents the extent to which an individual believes that he or she possesses traits associated with traditional gender stereotypes (Bem's Sex Role Inventory, 1981; Wood \& Eagly, 2010).

People differ in the extent to which they incorporate gender roles into their self-concepts. Two independent dimensions of gender identity arise: masculinity, or beliefs about the extent to which one possesses traits associated with males (e.g., dominance, independence, ambition) and femininity, or beliefs 
about the extent to which one possesses traits associated with females (e.g., sensitivity to the needs of others, compassion, understanding) (Eddleston \& Powell, 2008; Wood \& Eagly, 2010). Women are socialized to identify with attributes that are considered feminine, and men are socialized to identify with attributes that are considered masculine (Bem's Sex Role Inventory, 1981). As such, male entrepreneurs are more likely to exhibit a stereotypical masculine orientation and female entrepreneurs are most likely to exhibit a stereotypical feminine orientation (Bird \& Brush, 2002).

People use their gender identity as a standard against which to regulate their behavior. Entrepreneurs who have a masculine self-concept involving independence and dominance might regulate their behavior by, for example, seeking opportunities for high growth and financial rewards from their businesses (Bird \& Brush, 2002). Eddleston and Powell (2008) in their survey of entrepreneurial alumni show that women entrepreneurs value different sources of career satisfaction than men. Male entrepreneurs preferred satisfiers associated with status attainment (e.g., earning a lot of money, having high prestige and social status, being in a leadership role, leading a large, rapidly growing enterprise) whereas female entrepreneurs preferred satisfiers associated with employee relationships (e.g., working with friendly and congenial people, having supportive employees, working as part of a team) and making a contribution to society. Nevertheless, the study presents no evidence on whether business owners' masculinity relates more strongly to growth intentions compared to business owners' femininity.

\section{Boundary Conditions: The Role of Independent Self-Construal}

Research provides convincing evidence that the concept of entrepreneurial activity is gender-biased: Entrepreneurship is often depicted as a form of masculinity and the terms "entrepreneur" and "male" have tended to become interchangeable (Ahl \& Marlow 2012; Gupta et al., 2009). This supports a hierarchical valuation in which the masculine is prioritized over the feminine and the characteristics of successful business owners (i.e., proactivity, need for achievement, risk taking competiveness, confidence) are stereotypically perceived to be masculine (Ahl, 2006; Bird \& Brush, 2002; Coleman, 2016). Moreover, the behavior of women involved in entrepreneurial activity is defined and evaluated according to the standards of an invisible masculine norm (Lewis, 2006). Women entrepreneurs have to manage different identities simultaneously which can result in conflict (Ahl, 2006; Bird \& Brush, 2002).

Accordingly, to be recognized as credible actors within entrepreneurship, women have to learn the delicate balance between adopting a credible entrepreneurial identity which reflects masculinized norms but without denying ascribed femininity (Ahl \& Marlow 2012). Thus, one important question raised is how women entrepreneurs' growth intentions are formed despite the mixed messages they receive from a process that broadly emphasizes masculinity but also expects females specifically to care for and nurturing children or maintaining the household, or be supportive, that is, interdependent and connected with others. Building on the cultural dimensions of individualism and collectivism Cross and Madson (1997) propose additional facets of gender identity reflecting investment in an individual versus social sense of self. The authors define masculine identity as an independent sense of self and feminine identity as an interdependent sense of self.

Specifically, independent and interdependent self-construals represent one of the most fundamental and overarching schemata of an individual's selfhood (Markus \& Kitayama, 1991; Oyserman et al., 2002). Selfconstrual refers to the way an individual understands oneself in relation to other people. A person possessing an independent self-construal views one's self as separate from others, aiming to maintain a sense of autonomy and uniqueness. A person possessing an interdependent self-construal view one's self as connected to others, where self-presentations are woven together with representations of close others and social context (Markus \& Kitayama, 1991; Oyserman et al., 2002). Men are more likely to be likely to be socialized to construct an independent self-construal and develop a social self that is marked by placing primary emphasis on values of individual needs, autonomy, and self-fulfillment. Women are more likely to be socialized to construct an interdependent self-construal and develop a social self that is marked by the motivation to be connected with particular relationships or contexts (Cross \& Madson, 1997; Oyserman et al., 2002; Singelis, 1994). 
There is limited research to date that has assessed the relationship between self-construal and entrepreneurship. Recent empirical research provides evidence for the moderating role of individual-level self-construal in cognitive models of intention to start a new business (Siu \& Lo, 2013; Zampetakis et al., 2015). For instance, Siu and Lo (2013), using a sample of students from China and Hong Kong, found that the strength of perceived social norms in predicting entrepreneurial intention was dependent on interdependent self-construal. Zampetakis et al. (2015) using a sample of students from Greece found that independent self-construal was related to attitudes towards entrepreneurship and moderated relationships between attitudes and entrepreneurial intentions.

As noted above, in order for women to gain legitimacy as entrepreneurs, they are encouraged to adopt and reproduce attitudes and behaviors which are in fact reproductions of what men do and what men are (Ahl \& Marlow, 2012). However, some evidence suggests that when women emulate the behavior of the idealized male, there is evidence of misfit. For instance, Kerfoot and Miller (2010) evaluated the results of a training program for potential business owners aiming to encourage more women to start their own ventures. The program established men as natural entrepreneurs rendering women as outsiders, and the only hope for entry in the entrepreneurial process was by learning how to emulate the behavior of the idealized male. After undertaking the course, many of the potential female business owners were actively discouraged from starting new ventures. It became evident that they did not "fit" the masculinized image of an entrepreneur (Kerfoot \& Miller, 2010).

This suggests that when cues or symbols in the environment make independent self-construal more accessible (Hong et al., 2000; Oyserman \& Sorensen, 2009) (i.e., symbols of individual freedom, autonomy, personal fulfillment, and separation), then feminine entrepreneurs may have less need for growing their ventures because of the conflict inherent in the relation between independent self-construal and femininity. Specifically, individuals who see themselves as higher in femininity are more likely to consider themselves as a member of a group. This in turn prompts a cognitive style that is concerned with the negative consequences of behavior (that is business growth) and avoiding potential failure (Oyserman \& Sorensen, 2009). Thus, we expect that the interaction between independent self-construal and femininity for the prediction of growth to be negative.

\section{Overview of Study Aims and Hypotheses}

The purpose of the present study was to examine differences in the process of business growth of established firms, between male and female entrepreneurs. We focused on a specific aspect of firm growth - the intention of the entrepreneur - and whether and how biological sex, gender identity, and independent self-construal affect growth intentions.

Specifically, the study aimed firstly to determine whether and the extent to which biological sex is associated with gender identity and entrepreneurs' business growth intentions. We expected that the correlation between entrepreneurs' sex and gender identity to be strong such that male entrepreneurs are most likely to exhibit a stereotypical masculine orientation and female entrepreneurs are most likely to exhibit a stereotypical feminine orientation. Secondly, we aimed to test whether, and the extent to which, gender identity mediates biological sex effects on business growth intentions. We expected that gender identity would at least partially mediate biological sex effects on growth intentions; we expected the effect of masculinity on growth intentions to be stronger compared to the effect of femininity. Finally, we examined the moderating influences of independent self-construal that constitute boundary conditions of the theory. We expected that independent self-construal would moderate the relationship between sex and gender identity and between gender identity and growth intentions. To our knowledge the present study is one of the first to examine within-culture individual-level cultural orientations as antecedents to business growth intentions in some depth.

We conducted a moderated mediation analysis (Hayes, 2013) in order to clearly distinguish the mediatory power of gender identity and the moderating role of independent self-construal while controlling for both firm-level and individual-level variables. 


\section{CONCLUSION}

The findings of the present study make it clear that business growth intention is a complex phenomenon that may be influenced by gender. Our results indicate that gender identity mediates the influence of sex on business growth intentions and the mediation effects are contingent on entrepreneurs' independent selfconstrual. Thus, women make decisions related to the growth of their businesses using a different process than men do. Our results reinforce the claims that it is valuable to incorporate a feminine perspective when studying the factors influencing entrepreneurs' growth intentions in established business.

\section{APPENDIX}

FIGURE 1

REPRESENTATION OF THE HYPOTHESIZED THEORETICAL MODEL

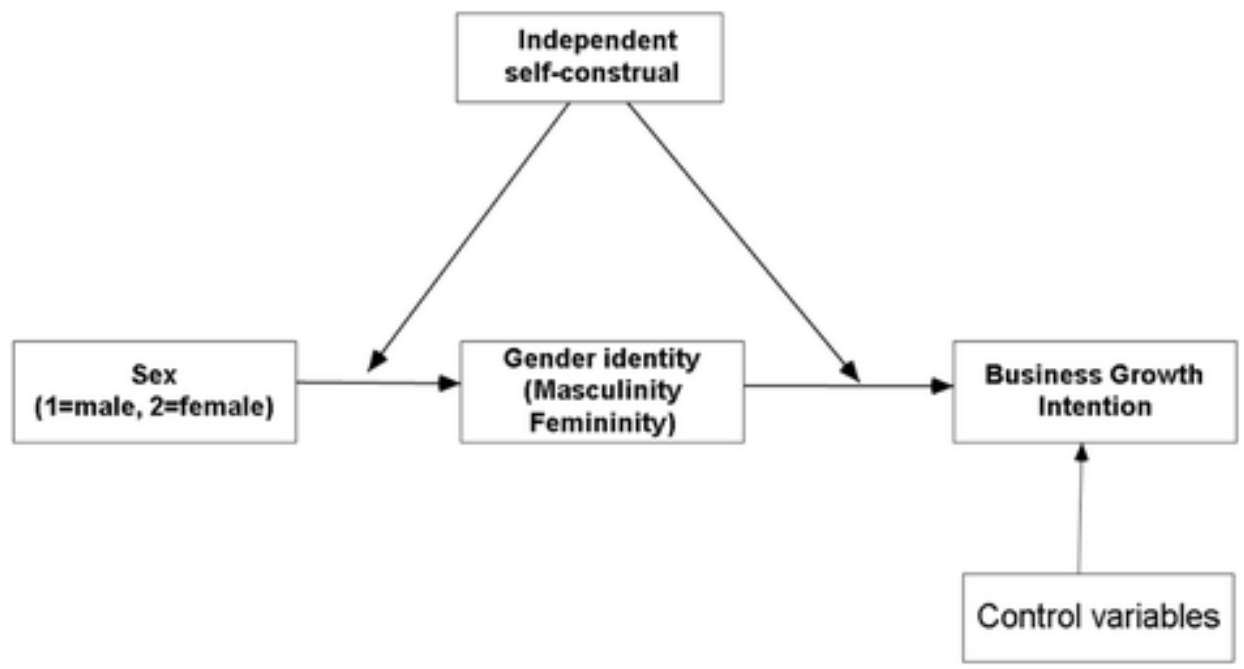




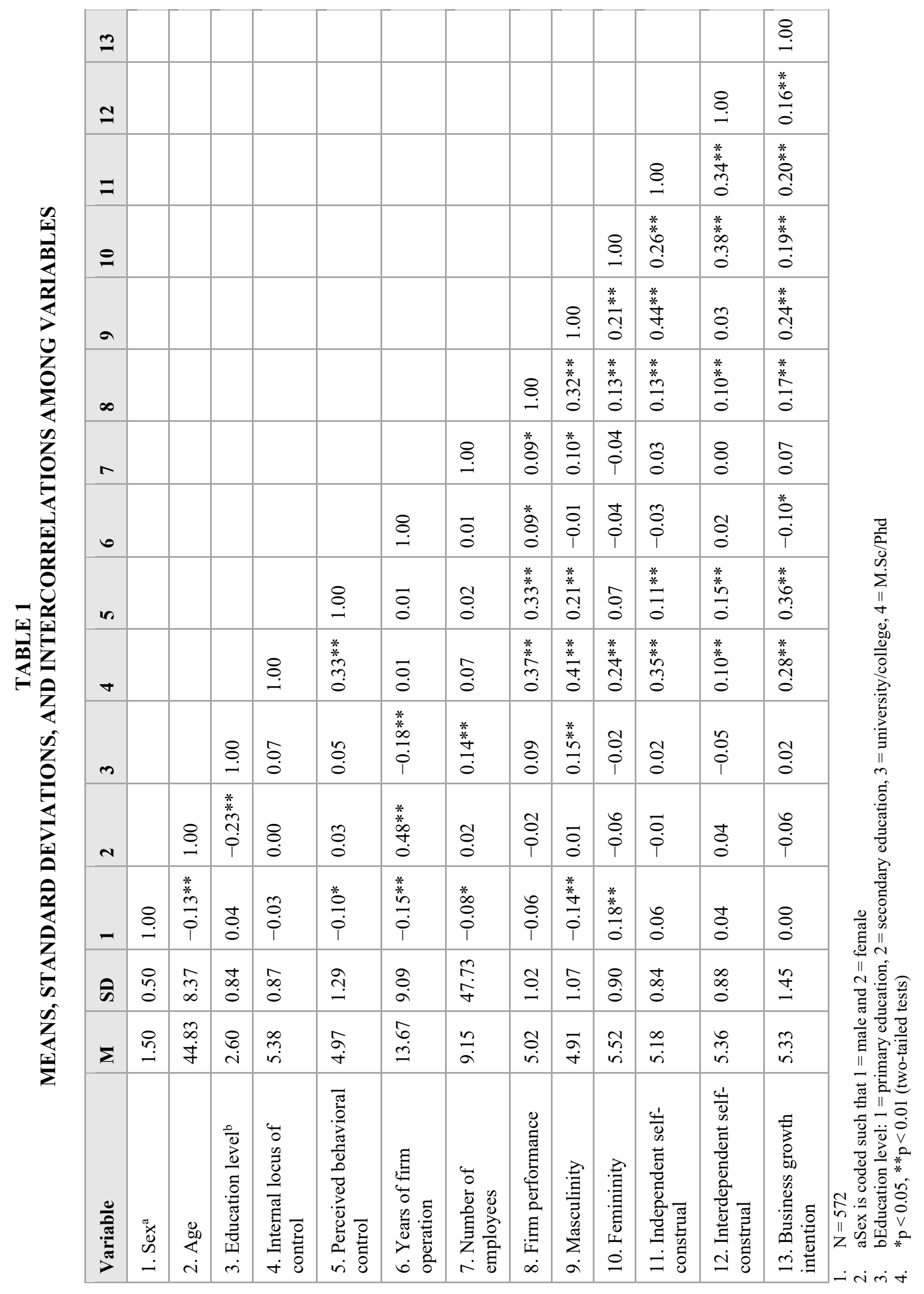

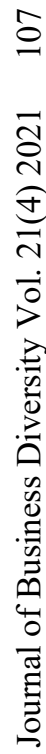


TABLE 2

TEST OF FIRST AND SECOND STAGE MODERATED MEDIATION MODEL

\begin{tabular}{|c|c|c|c|}
\hline \multirow[b]{2}{*}{ Predictor } & \multicolumn{2}{|c|}{ First stage moderation } & \multirow{2}{*}{$\begin{array}{l}\text { Second stage } \\
\text { moderation }\end{array}$} \\
\hline & Femininity & Masculinity & \\
\hline & B & $\boldsymbol{B}$ & B \\
\hline Constant & $\begin{array}{l}0.48 * *(-0.72 \text { to } \\
-0.23)\end{array}$ & $\begin{array}{l}0.49 * *(0.26 \text { to } \\
0.72)\end{array}$ & $0.07^{\text {ns }}(-0.23$ to 0.25$)$ \\
\hline $\operatorname{Sex}^{\mathrm{a}}$ & $\begin{array}{l}0.32 * *(0.16 \text { to } \\
0.48)\end{array}$ & $\begin{array}{l}-0.32 * *(-0.47 \text { to } \\
-0.18)\end{array}$ & $0.02^{\mathrm{ns}}(-0.13$ to 0.17$)$ \\
\hline Age & & & $0.001^{\mathrm{ns}}(-0.09$ to 0.08$)$ \\
\hline Education level $^{\mathrm{b}}$ & & & $-0.06^{\mathrm{ns}}(-0.14$ to 0.02$)$ \\
\hline $\begin{array}{l}\text { Entrepreneur's internal locus of } \\
\text { control }\end{array}$ & & & $0.08^{\text {ns }}(-0.02$ to 0.17$)$ \\
\hline $\begin{array}{l}\text { Entrepreneur's perceived } \\
\text { behavioral control }\end{array}$ & & & $0.31 * *(0.23$ to 0.38$)$ \\
\hline Firm age & & & $\begin{array}{l}-0.09 *(-0.18 \text { to } \\
-0.03)\end{array}$ \\
\hline Number of employees & & & $0.05^{\mathrm{ns}}(-0.02$ to 0.13$)$ \\
\hline Firm performance & & & $-0.02^{\mathrm{ns}}(-0.10$ to 0.06$)$ \\
\hline Femininity & & & $0.10 * *(0.09$ to 0.18$)$ \\
\hline Masculinity & & & $0.10 * *(0.08$ to 0.19$)$ \\
\hline Independent self-construal (IND) & $\begin{array}{l}0.54 * *(0.29 \text { to } \\
0.80)\end{array}$ & $\begin{array}{l}0.44 * *(0.20 \text { to } \\
0.68)\end{array}$ & $0.05^{\text {ns }}(-0.03$ to 0.14$)$ \\
\hline Sex X IND & $\begin{array}{l}-0.19 *(-0.35 \text { to } \\
-0.11)\end{array}$ & $\begin{array}{l}0.03^{\text {ns }}(-0.14 \text { to } \\
0.14)\end{array}$ & \\
\hline IND $X$ femininity & & & $\begin{array}{l}-0.09 *(-0.17 \text { to } \\
-0.05)\end{array}$ \\
\hline IND X masculinity & & & $-0.03^{\text {ns }}(-0.09$ to 0.03$)$ \\
\hline
\end{tabular}

1. The upper and lower bounds of the $95 \%$ confidence interval (shown in parentheses) were based on the findings from a bootstrapping analysis using the percentile method

2. $\quad{ }^{\text {a}} \mathrm{Sex}$ is coded such that $1=$ male and $2=$ female

3. ${ }^{\mathrm{b}}$ Education level: 1 = primary education, $2=$ secondary education, $3=$ university $/$ college, $4=\mathrm{M} . \mathrm{Sc} / \mathrm{Phd}$

4. ${ }^{\mathrm{ns}}$ nonsignifiant

5. ${ }^{*} p<0.01,{ }^{* *} p<0.001$ (two-tailed tests) 


\section{FIGURE 2}

RELATIONSHIP BETWEEN ENTREPRENEURS' FEMININITY AND BUSINESS GROWTH INTENTIONS FOR DIFFERENT LEVELS OF THE MODERATOR VARIABLE

(INDEPENDENT SELF-CONSTRUAL): THE MEAN, ONE STANDARD

DEVIATION ABOVE THE MEAN (1SD), AND ONE STANDARD

DEVIATION BELOW THE MEAN (-1SD)

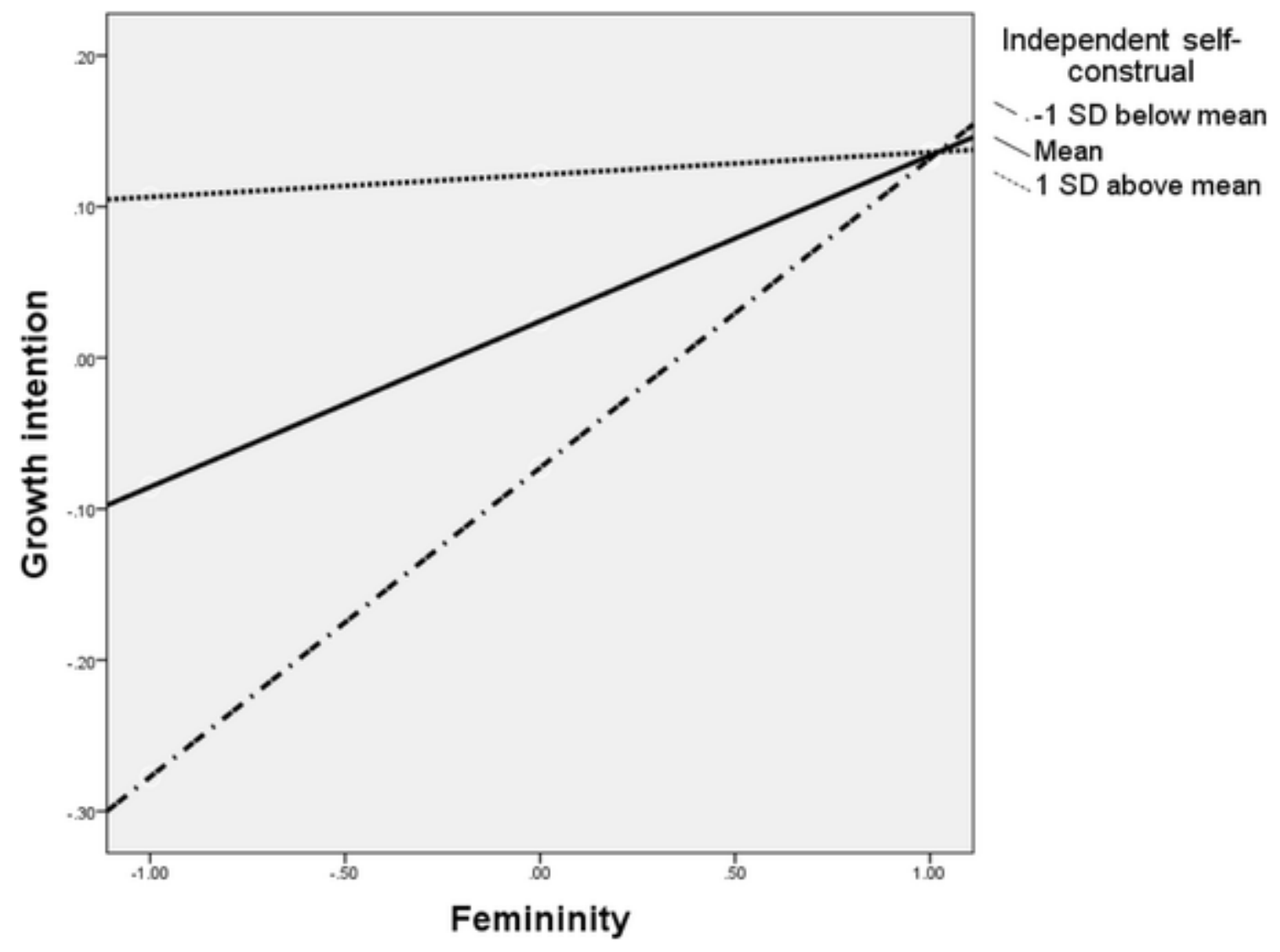

TABLE 3

CONDITIONAL INDIRECT EFFECT RESULTS OF BIOLOGICAL SEX ON BUSINESS GROWTH INTENTIONS AT VALUES OF INDEPENDENT SELF-CONSTRUAL

\begin{tabular}{|l|l|l|l|l|}
\hline \multirow{2}{*}{ Model } & Mediator & $\begin{array}{l}\text { Level } \\
\text { of IND }\end{array}$ & \multicolumn{2}{l|}{ Business growth intention } \\
\cline { 3 - 5 } & & & Estimate & $\begin{array}{l}\text { 95 \% Confidence } \\
\text { interval }\end{array}$ \\
\hline \multirow{2}{*}{$\begin{array}{l}\text { Biological sex (via gender identity) on } \\
\text { business growth intent }\end{array}$} & Femininity & -1 SD & 0.11 & 0.03 to 0.20 \\
\cline { 3 - 6 } & & Mean & 0.03 & 0.01 to 0.07 \\
\cline { 3 - 6 } & & +1 SD & 0.00 & -0.02 to 0.03 \\
\cline { 3 - 6 } & Masculinity & -1 SD & -0.04 & -0.10 to -0.01 \\
\cline { 3 - 6 } & & Mean & -0.03 & -0.08 to -0.008 \\
\hline
\end{tabular}




\begin{tabular}{|l|l|l|l|l|}
\hline & Mediator & $\begin{array}{l}\text { Level } \\
\text { of IND }\end{array}$ & \multicolumn{2}{|l|}{ Business growth intention } \\
\cline { 4 - 5 } & & & Estimate & $\begin{array}{l}\mathbf{9 5} \% \text { Confidence }^{\mathbf{a}} \\
\text { interval }^{\mathbf{b}}\end{array}$ \\
\hline & & +1 SD & -0.025 & -0.08 to 0.008 \\
\hline
\end{tabular}

1. $N=572$

2. $S E$ standard error, $I N D$ independent self-construal

3. ${ }^{\mathrm{a}}$ Control variables $=$ firm age, number of employees, firm performance, entrepreneur's age, education, internal locus of control, and perceived behavioral control

4. ${ }^{\mathrm{b} B i a s}$ corrected and accelerated confidence intervals are reported. Bootstrap sample size $=5000$

\section{REFERENCES}

Ahl, H. (2006). Why research on women entrepreneurs needs new directions. Entrepreneurship Theory \& Practice, 30(5), 595-621.

Ahl, H., \& Marlow, S. (2012). Exploring the dynamics of gender, feminism and entrepreneurship: Advancing debate to escape a dead end? Organization Science, 19(5), 543-562.

Ajzen, I. (1991). The theory of planned behavior. Organizational Behavior and Human Decision Processes, 50, 179-211.

Arbuckle, J.L. (2006). AMOS 7.0. user guide. Chicago: SmallWaters Corporation.

Baumeister, R.F., Bratslavsky, E., Muraven, M., \& Tice, D.M. (1998). Ego depletion: Is the active self a limited resource? Journal of Personality and Social Psychology, 74(5), 1252-1265.

Bem's Sex Role Inventory. (1981). Gender schema theory: A cognitive account of sex typing. Psychological Review, 88(4), 354-364. doi:10.1037/0033-295x.88.4.354

Bird, B., \& Brush, C. (2002). A gendered perspective on organizational creation. Entrepreneurship Theory and Practice, 26(3), 41-66.

Bjursell, C., \& Bäckvall, L. (2011). Family business women in media discourse: The business role and the mother role. Journal of Family Business Management, 1, 154-173.

Bulanova, O., Isaksen, E.J., \& Kolvereid, L. (2016). Growth aspirations among women entrepreneurs in high growth firms. Baltic Journal of Management, 11(2), 187-206.

Carayannis, E.G., Samara, E.T., \& Bakouros, Y.L. (2015). Innovation and entrepreneurship: Theory, policy and practice. Heidelberg: Springer.

Carver, L.F., Vafaei, A., Guerra, R., Freire, A., \& Phillips, S.P. (2013). Gender differences: Examination of the 12-item Bem Sex Role Inventory (BSRI-12) in an older Brazilian population. PLoS ONE, 8(10), e76356. doi:10.1371/journal.pone.0076356

Cassar, G. (2006). Entrepreneur opportunity costs and intended venture growth. Journal of Business Venturing, 21(5), 610-632.

Chen, C.C., Greene, P.G., \& Crick, A. (1998). Does entrepreneurial self-efficacy distinguish entrepreneurs from managers? Journal of Business Venturing, 13(4), 295-316.

Cliff, J.E. (1998). Does one size fit all? Exploring the relationship between attitudes towards growth, gender, and business size. Journal of Business Venturing, 13(6), 523-542.

Cohen, J., Cohen, P., West, S.G., \& Aiken, L.S. (2003). Applied multiple regression/correlation analysis for the behavior sciences. Mahwah: Lawrence Erlbaum Associates.

Coleman, S. (2016). Gender, entrepreneurship, and firm performance: Recent research and considerations of context. In M.L. Connerley \& J. Wu (Eds.), Handbook on well-being of working women (pp. 375-391). Dordrecht: Springer Science.

Cross, S.E., \& Madson, L. (1997). Models of the self: self-construals and gender. Psychological Bulletin, 122(1), 5-37. 
Davis, A.E., \& Shaver, K.G. (2012). Understanding gendered variations in business growth intentions across the life course. Entrepreneurship Theory and Practice, 36(3), 495-515. doi:10.1111/j.1540-6520.2012.00508.x

Delmar, F., \& Wiklund, J. (2008). The effect of small business managers' growth motivation on firm growth: A longitudinal study. Entrepreneurship Theory and Practice, 32(3), 437-457.

Dutta, D.K., \& Thornhill, S.1. (2008). The evolution of growth intentions: Toward a cognition-based mode. Journal of Business Venturing, 23(3), 307-332.

Eddleston, K.A., \& Powell, G.N. (2008). The role of gender identity in explaining sex differences in business owners' career satisfier preferences. Journal of Business Venturing, 23(2), 244-256.

Edelman, L.F., Brush, C.G., Manolova, T.S., \& Greene, P.G. (2010). Start-up motivations and growth intentions of minority nascent entrepreneurs. Journal of Small Business Management, 48(2), 174196.

Farmer, S.M., Yao, X., \& Kung-Mcintyre, K. (2011). The behavioral impact of entrepreneur identity aspiration and prior entrepreneurial experience. Entrepreneurship Theory and Practice, 35(2), 245-273.

Fauchart, E., \& Gruber, M. (2011). Darwinians, communitarians, and missionaries: The role of founder identity in entrepreneurship. Academy of Management Journal, 54(5), 935-957.

Fernández, I., Paez, D., \& González, J.L. (2005). Independent and interdependent self-construals and socio-cultural factors in 29 nations. Revue Internationale de Psychologie Sociale, 18(1), 35-63.

Gupta, V.K., Turban, D.B., Wasti, S.A., \& Sikdar, A. (2009). The role of gender stereotypes in perceptions of entrepreneurs and intentions to become an entrepreneur. Entrepreneurship Theory and Practice, 33(2), 397-417.

Haltiwanger, J.C., Lane, J.I., \& Spletzer, J.R. (1999). Productivity differences across employers: The roles of employer size, age, and human capital. The American Economic Review, 89(2), 94-98.

Hayes, A.F. (2013). Introduction to mediation, moderation, and conditional process analysis: A regression-based approach. New York: The Guilford Press.

Henry, C., Foss, L., \& Ahl, H. (2016). Gender and entrepreneurship research: A review of methodological approaches. International Small Business Journal, 34(3), 217-341.

Hoang, H., \& Gimeno, J. (2010). Becoming a founder: How founder role identity affects entrepreneurial transitions and persistence in founding. Journal of Business Venturing, 25(1), 41-53.

Hong, Y.Y., Morris, M.W., Chiu, C.Y., \& Benet-Martinez, V. (2000). Multicultural minds: A dynamic constructivist approach to culture and cognition. American Psychologist, 55(7), 709-720.

Ireland, R.D., \& Webb, J.W. (2007). A cross-disciplinary exploration of entrepreneurship research. Journal of Management, 33(6), 891-927.

Jennings, J.E., \& Brush, C.G. (2013). Research on women entrepreneurs: Challenges to (and from) the broader entrepreneurship literature? Academy of Management Annals, 7(1), 661-713.

Kerfoot, D., \& Miller, C. (2010). Organizing entrepreneurship? Women's invisibility in self-employment. In P. Lewis \& S. Ruth (Eds.), Revealing and concealing gender (pp. 100-123). UK: Palgrave Macmillan.

Krueger, N.F., Reilly, M.D., \& Carsrud, A.L. (2000). Competing models of entrepreneurial intentions. Journal of Business Venturing, 15(5), 411-432.

Lewis, P. (2006). The quest for invisibility: Female entrepreneurs and the masculine norm of entrepreneurship. Gender, Work \& Organization, 13(5), 453-469.

Linan, F., \& Chen, Y.W. (2009). Development and cross-cultural application of a specific instrument to measure entrepreneurial intentions. Entrepreneurship Theory and Practice, 33(3), 593-617.

Lindberg, M. (2012). A striking pattern co-construction of innovation, men and masculinity in Sweden's innovation policy. In S. Andersson, K. Berglund, E. Gunnarsson, \& E. Sundin (Eds.), Promoting innovation: Policies, practices and procedures. Stockholm: VINNOVA/Swedish Governmental Agency for Innovation System.

Markus, H.R., \& Kitayama, S. (1991). Culture and the self: Implications for cognition, emotion, and motivation. Psychological Review, 98(2), 224-253. 
Menzies, T.V., Diochon, M., \& Gasse, Y. (2004). Examining venture-related myths concerning women entrepreneurs. Journal of Developmental Entrepreneurship, 9, 89-107.

Miller, C.C., Washburn, N.T., \& Glick, W.H. (2013). Perspective-the myth of firm performance. Organization Science, 24(3), 948-964.

Mueller, S.L. (2004). Gender gaps in potential for entrepreneurship across countries and cultures. Journal of Developmental Entrepreneurship, 9(3), 199-220.

Oppenheimer, D.M., Meyvis, T., \& Davidenko, N. (2009). Instructional manipulation checks: Detecting satisficing to increase statistical power. Journal of Experimental Social Psychology, 45(4), 867872.

Oyserman, D., \& Sorensen, N. (2009). Understanding cultural syndrome effects on what and how we think: A situated cognition model. In R.S. Wyer, C. Chi-yue, \& Y.Y. Hong (Eds.), Understanding culture: Theory, research and application (pp. 25-52). New York: Taylor \& Francis Group.

Oyserman, D., Coon, H.M., \& Kemmelmeier, M. (2002). Rethinking individualism and collectivism: Evaluation of theoretical assumptions and meta-analyses. Psychological Bulletin, 128, 3-72.

Pettersson, K., \& Lindberg, M. (2013). Paradoxical spaces of feminist resistance: Mapping the margin to the masculinist innovation discourse. International Journal of Gender and Entrepreneurship, 5(3), 323-341.

Podsakoff, P.M., MacKenzie, S.B., Lee, J.Y., \& Podsakoff, N.P. (2003). Common method biases in behavioral research: A critical review of the literature and recommended remedies. Journal of Applied Psychology, 85(5), 879-903.

Preacher, K.J., \& Kelley, K. (2011). Effect size measures for mediation models: Quantitative strategies for communicating indirect effects. Psychological Methods, 16(2), 93-115.

Rauch, A., \& Frese, M. (2000). Psychological approaches to entrepreneurial success. A general model and an overview of findings. In C.L. Cooper \& I.T. Robertson (Eds.), International Review of Industrial and Organizational Psychology (pp. 101-142). Chichester: Wiley.

Sadler-Smith, E., Hampson, Y., Chaston, I., \& Badger, B. (2003). Managerial behavior, entrepreneurial style, and small firm performance. Journal of Small Business Management, 41(1), 47-67.

Savino, D.M. (2016). Frederick Winslow Taylor and His Lasting Legacy of Functional Leadership Competence. Journal of Leadership, Accountability and Ethics, 13(1). Retrieved from https://articlegateway.com/index.php/JLAE/article/view/1926

Shane, S. (2009). Why encouraging more people to become entrepreneurs is bad public policy. Small Business Economics, 33(2), 141-149.

Shepherd, D., \& Haynie, J.M. (2009). Family business, identity conflict, and an expedited entrepreneurial process: A process of resolving identity conflict. Entrepreneurship Theory and Practice, 33(6), $1245-1264$.

Shook, C.L., Ketchen, D.J.J., Hult, G.T.M., \& Kacmar, K.M. (2004). An assessment of the use of structural equation models in strategic management research. Strategic Management Journal, 25, 397-404.

Shrout, P.E., \& Bolger, N. (2002). Mediation in experimental and non-experimental studies: New procedures and recommendations. Psychological Methods, 4, 422-445.

Singelis, T.M. (1994). The measurement of independent and interdependent self-construals. Personality and Social Psychology Bulletin, 20, 580-591.

Siu, W.S., \& Lo, E.S.C. (2013). Cultural contingency in the cognitive model of entrepreneurial intention. Entrepreneurship Theory and Practice, 37, 147-173.

Stam, E., Suddle, K., Hessels, J., \& van Stel, A. (2009). High-growth entrepreneurs, public policies and economic growth. In J. Leitao \& R. Baptista (Eds.), Public policies for fostering entrepreneurship: A European perspective (pp. 91-110). New York: Springer.

Tang, L., \& Koveos, P.E. (2004). Venture entrepreneurship, innovation entrepreneurship and economic growth. Journal of Developmental Entrepreneurship, 3, 161-171. 
Vafaei, A., Alvarado, B., Tomás, C., Muro, C., Martinez, B., \& Zunzunegui, M.V. (2014). The validity of the 12-item Bem Sex Role Inventory in older Spanish population: An examination of the androgyny model. Archives of Gerontology and Geriatrics, 59(2), 257-263.

Van Praag, C.M., \& Versloot, P. (2007). What is the value of entrepreneurship? A review of recent research. Small Business Economics, 29(4), 351-382.

Venugopal, V. (2016). Investigating women's intentions for entrepreneurial growth. International Journal of Gender and Entrepreneurship, 8(1), 2-27.

Wiklund, J., \& Shepherd, D. (2003). Aspiring for, and achieving growth: The moderating role of resources and opportunities. Journal of Management Studies, 40(8), 1919-1941.

Wiklund, J., Davidsson, P., \& Delmar, F. (2003). What do they think and feel about growth? An expectancy-value approach to small business managers' attitudes toward growth.

Entrepreneurship Theory and Practice, 27(3), 247-270.

Wood, W., \& Eagly, A.H. (2010). Gender. In S.T. Fiske, D.T. Gilbert, \& G. Lindzey (Eds.), Handbook of social psychology (5th ed., Vol. 1, pp. 629-667). Hoboken: Wiley.

Zampetakis, L.A., Lerakis, M., Kafetsios, K., \& Moustakis, V. (2015). Investigating the role of selfconstrual in the formation of entrepreneurial intentions. Frontiers in Psychology - Organizational Psychology, 6(1085). doi:10.3389/fpsyg.2015.01085

Zhao, X., Lynch, J.G., \& Chen, Q. (2010). Reconsidering Baron and Kenny: Myths and truths about mediation analysis. Journal of Consumer Research, 37(2), 197-206.

\section{TRANSLATED VERSION: SPANISH}

Below is a rough translation of the insights presented above. This was done to give a general understanding of the ideas presented in the paper. Please excuse any grammatical mistakes and do not hold the original authors responsible for these mistakes.

\section{VERSION TRADUCIDA: ESPAÑOL}

A continuación se muestra una traducción aproximada de las ideas presentadas anteriormente. Esto se hizo para dar una comprensión general de las ideas presentadas en el documento. Por favor, disculpe cualquier error gramatical y no responsabilite a los autores originales de estos errores.

\section{INTRODUCCIÓN}

El desarrollo y crecimiento de los negocios existentes es un proceso con muchos beneficios sociales, incluida la creación de empleo y riqueza y el avance de la innovación (Tang \& Koveos, 2004; Van Praag $\&$ Versloot, 2007). La decisión de los empresarios para hacer crecer su negocio es compleja, no es lineal ni depende de un número limitado de factores (Miller et al. 2013). Los empresarios no solo tienen que tomar decisiones importantes para diferentes tareas que son de naturaleza diferente, sino que también deben asegurarse de que esas decisiones sean las correctas no solo para su negocio sino también para ellos mismos. Mientras que algunos empresarios se complacen en ser autónomos a pequeña escala, otros tienen aspiraciones de crecimiento para sus empresas (Shane, 2009).

La investigación delinea claramente que las empresas propiedad de mujeres tienden a tener niveles más bajos de crecimiento y siguen siendo más pequeñas que las empresas propiedad de hombres (Cliff, 1998; Coleman, 2016; Davis \& Shaver, 2012), sugiriendo la existencia de una brecha entre hombres y mujeres en el emprendimiento; este es un tema que está atrayendo cada vez más atención académica (Coleman, 2016). Sin embargo, gran parte de esa investigación ha investigado las diferencias en los niveles de actividad inicial entre hombres y mujeres (ver Jennings y Brush, 2013). Poca investigación ha examinado las aspiraciones de crecimiento de las empresas lideradas por mujeres. En comparación con los empresarios varones, las empresarias tienden a perseguir objetivos no económicos, como equilibrar los roles laborales y familiares, y tienen preferencias por las relaciones con los empleados y los satisfactores de la sociedad, 
lo que a su vez puede restar rendimiento económico o crecimiento (Eddleston y Powell, 2008; Jennings \& Brush, 2013).

Como señalan Henry et al. (2016), las representaciones normativas del emprendimiento están dominadas por la masculinidad. Esto margina a las mujeres empresarias y las hace invisibles. Los autores señalan que si bien la conciencia de que el género (masculino y femenino) se construye socialmente y corresponde a un conjunto aprendido de comportamientos que no se refiere simplemente al sexo biológico (hombre / hombre y mujer / mujer) del empresario parece generalizada entre los académicos, varios temas perjudiciales relacionados con las mujeres son persistentes. Por ejemplo, muchos académicos utilizan las normas masculinas para juzgar las actividades de las mujeres simplemente comparando hombres y mujeres, con poca o ninguna atención prestada a las construcciones de género (Ahl, 2006; Lewis, 2006). Sin embargo, esta práctica descuida el hecho de que los aspectos masculinos y femeninos se incorporarán al proceso de crecimiento empresarial tanto para hombres como para mujeres. Es decir, el género podría ser un aspecto vital de la intención de crecimiento empresarial.

En conjunto, hasta la fecha, la investigación no proporciona explicaciones concluyentes para la variación en la trayectoria de crecimiento que los hombres y las mujeres desean que siga su empresa (es decir, su intención de crecimiento) (Bulanova et al. 2016; Dutta \& Thornhill, 2008). La intención de crecimiento se considera una característica esencial del comportamiento empresarial y un elemento clave para comprender el desarrollo y el crecimiento de las empresas (Sadler-Smith et al. 2003). Además, las intenciones han demostrado ser el mejor predictor del comportamiento planificado (Ajzen, 1991), especialmente si el fenómeno implica retrasos impredecibles, planificación y un alto grado de procesamiento cognitivo, como el crecimiento empresarial (Krueger et al. 2000). Las intenciones de crecimiento se consideran un predictor clave del crecimiento real del negocio (Delmar \& Wiklund, 2008; Wiklund \& Shepherd, 2003).

Evidencia considerable sugiere que la identidad de un individuo (o concepto de sí mismo) en general (Fauchart \& Gruber, 2011; Hoang \& Gimeno, 2010) y la identidad de género (o roles de género relacionados) en particular (Eddleston \& Powell, 2008; Gupta et al., 2009) pueden contribuir a diferencias sustanciales entre hombres y mujeres en el comportamiento empresarial. Las personas tienden a diferir en la medida en que incorporan los roles de género en sus autoconci conceptos.

En el presente estudio, proponemos que la identidad de género de los empresarios o la medida en que los empresarios poseen rasgos asociados con los estereotipos de género tradicionales (Bem's Sex Role Inventory, 1981; Mueller, 2004; Wood \& Eagly, 2010) es un importante mecanismo cognitivo que relaciona el sexo de los emprendedores con las intenciones de crecimiento empresarial. Argumentamos que la identidad de género da forma a la forma en que los empresarios se ven a sí mismos, cómo entienden el mundo que los rodea y se acercan a otras personas, pero también lo que pretenden lograr en el futuro (Eddleston y Powell, 2008; Hoang \& Gimeno, 2010).

Sin embargo, los mecanismos por los cuales el sexo y la identidad de género ejercen influencia sobre las intenciones de crecimiento de los empresarios y las influencias moderadoras que constituyen las condiciones límite de la teoría necesitan una mayor investigación. La autointerpretación es un moderador potencial de la relación entre el género, la identidad de género y las intenciones de crecimiento. La autoconstrucción se refiere a los pensamientos, sentimientos y acciones culturalmente contingentes de los individuos que se refieren a la comprensión del yo como conectado a los demás (autoconstrucción interdependiente) o distinto de los demás (autoconstrucción independiente) (Markus y Kitayama, 1991). La exposición de los individuos a ciertas condiciones puede aumentar la probabilidad de que exhiban tendencias independientes (individualistas) o interdependientes (colectivistas) (Hong et al., 2000). Los conceptos de "emprendedor" y "emprendimiento" se consideran de género masculino (Ahl, 2006; Lewis, 2006). Las señales o símbolos en el entorno que los empresarios utilizan para operar su negocio podrían hacer que la autoconstrucción independiente sea más accesible (Hong et al., 2000), ya que la identidad masculina destaca los atributos únicos de los individuos (Cross \& Madson, 1997). Una consideración sistemática de los posibles efectos moderadores de la autointerpretación contribuirá a una comprensión más completa de las condiciones en las que es más probable que se mantengan los efectos del sexo en las intenciones de crecimiento. 
En resumen, el objetivo de este artículo es profundizar en la comprensión de los factores que influyen en la motivación del crecimiento empresarial en las empresas establecidas. Con este fin, uno de los principales objetivos del presente estudio es examinar la relación entre el sexo de los empresarios, la identidad de género y las intenciones de crecimiento de los propietarios de negocios de sus empresas establecidas. Razonamos que una exploración de las intenciones de crecimiento de los dueños de negocios de empresas establecidas podría ayudar a explicar las diferencias de sexo en la decisión de crecer. Un segundo propósito importante del presente estudio es centrarse en la autointerpretación de los empresarios como un moderador potencial de la relación entre el sexo y la identidad de género y entre la identidad de género y la intención de crecimiento empresarial (ver modelo conceptual en la Fig. 1). Hasta donde sabemos, hasta la fecha, ha habido poca investigación que explique la influencia de estos dominios y la intención de los empresarios para el crecimiento del negocio establecido. Sin embargo, esto es importante teniendo en cuenta que las personas comienzan y operan sus empresas por una variedad de razones distintas al crecimiento o la maximización de los rendimientos económicos (Wiklund et al. 2003).

El documento comienza con un análisis de las intenciones de crecimiento del negocio; esto es seguido por una evaluación de la influencia de la identidad de género en las intenciones de crecimiento y el papel moderador de la autoconstrucción. A continuación, describimos nuestra muestra, métodos de investigación y técnicas de análisis de datos que incluyen ANOVA y análisis de mediación moderada. Finalmente, discutimos los resultados del análisis empírico y las limitaciones de la investigación, presentamos la implicación práctica de los resultados de la investigación y proponemos áreas para futuras investigaciones.

\section{Intención de Crecimiento Eidentidad de Género de los Emprendedores}

El crecimiento empresarial es un fenómeno multidimensional y complejo que implica una cuidadosa planificación y pensamiento por parte del empresario. Como tal, el crecimiento del negocio puede considerarse un comportamiento intencional deliberado y planificado y, en consecuencia, aplicable a los modelos de intención (Ajzen, 1991; Krueger et al., 2000). Las intenciones de crecimiento son fundamentales para la comprensión del crecimiento empresarial, ya que son el primer paso en el proceso e involucran un elemento intencional con pasos específicos para alcanzar la meta (Delmar y Wiklund, 2008; Venugopal, 2016). Las intenciones se han identificado como un predictor clave del comportamiento real, a través de una amplia gama de comportamientos diferentes, incluida la creación de negocios (Delmar y Wiklund, 2008; Wiklund \& Shepherd, 2003). Además, la investigación proporciona evidencia de que la proporción de empresarios con intenciones de crecimiento en la población es un predictor significativo del crecimiento económico en comparación con las tasas de autoempleo o las tasas generales de puesta en marcha (Stam et al., 2009).

Hasta la fecha, la investigación sobre las intenciones de crecimiento de los empresarios ha producido resultados mixtos con respecto a las diferencias empíricas entre hombres y mujeres y no todos los académicos están de acuerdo en que las mujeres y los hombres difieren en su intención general de crecimiento empresarial (Cassar, 2006; Davis \& Shaver, 2012). Por ejemplo, Cassar (2006) encontró que las mujeres empresarias tenían estimaciones más bajas de ingresos futuros que los hombres. Sin embargo, Menzies et al. (2004), en un estudio realizado en Canadá, no encontraron diferencias estadísticamente significativas entre mujeres y hombres en sus preferencias expresadas por el crecimiento sin restricciones. En el presente estudio, proponemos que los procesos de socialización de género pueden explicar las diferencias entre las intenciones de crecimiento empresarial de los empresarios masculinos y femeninos.

La investigación contemporánea en emprendimiento sugiere que decisiones como el crecimiento de las empresas establecidas están íntimamente entrelazadas con la identidad de los empresarios (Shepherd \& Haynie 2009). La identidad es un proceso social fluido que tiene una gama de significados conceptuales y roles teóricos asociados con ella (Fauchart \& Gruber, 2011; Hoang \& Gimeno, 2010). Puede verse como la representación de las personas de la internalización e incorporación de expectativas de comportamiento socialmente sostenidas. Además, la identidad puede considerarse como un concepto fundamental de puente entre lo individual y lo social (Hoang \& Gimeno, 2010). Los individuos se construyen socialmente a través de interacciones sociales y que adquieren a lo largo de su vida identidades sociales diversas y múltiples. En el caso del proceso empresarial, Ireland y Webb (2007) señalan en su revisión que para los empresarios, el 
proceso empresarial se basa e impulsa por identidades propias. Los modelos conceptuales recientes del papel de la identidad en el emprendimiento proponen fuertes vínculos entre el autoconcepto de los empresarios y las acciones y resultados empresariales (Shepherd \& Haynie 2009), pero hasta la fecha, la investigación empírica es limitada (Farmer et al. 2011). La identidad de género se considera un elemento esencial de una concepción multifaciente del yo que sitúa a los individuos dentro de las estructuras sociales (Eddleston \& Powell, 2008; Wood \& Eagly, 2010). El género es diferente del sexo (Ahl, 2006). Los investigadores han distinguido el sexo del género durante las décadas de 1970 y 1980 para distinguir entre las características biológicas de hombres y mujeres y los significados que las sociedades y los individuos atribuyen a las categorías masculinas y femeninas (Bem's Sex Role Inventory, 1981; Wood \& Eagly, 2010). A los individuos a lo largo del curso de la vida se les enseña (implícita o explícitamente) qué comportamientos y roles son deseables para hombres o mujeres en la sociedad. Como tal, la mayoría de las personas aceptan o internalizan los significados culturales asociados con sus significados sexuales. La identidad de género representa la medida en que un individuo cree que posee rasgos asociados con los estereotipos de género tradicionales (Bem's Sex Role Inventory, 1981; Wood \& Eagly, 2010).

Las personas difieren en la medida en que incorporan los roles de género en sus autoconci conceptos. Surgen dos dimensiones independientes de la identidad de género: la masculinidad, o las creencias sobre la medida en que uno posee rasgos asociados con los hombres (por ejemplo, dominación, independencia, ambición) y la feminidad, o creencias sobre la medida en que uno posee rasgos asociados con las mujeres (por ejemplo, sensibilidad a las necesidades de los demás, compasión, comprensión) (Eddleston y Powell, 2008; Wood \& Eagly, 2010). Las mujeres son socializadas para identificarse con atributos que se consideran femeninos, y los hombres son socializados para identificarse con atributos que se consideran masculinos (Bem's Sex Role Inventory, 1981). Como tal, los empresarios masculinos son más propensos a exhibir una orientación masculina estereotipada y las mujeres empresarias son más propensas a exhibir una orientación femenina estereotipada (Bird \& Brush, 2002).

Las personas usan su identidad de género como un estándar contra el cual regular su comportamiento. Los empresarios que tienen un autoconcepto masculino que implica independencia y dominio podrían regular su comportamiento, por ejemplo, buscando oportunidades de alto crecimiento y recompensas financieras de sus negocios (Bird \& Brush, 2002). Eddleston y Powell (2008) en su encuesta de ex alumnos emprendedores muestran que las mujeres empresarias valoran diferentes fuentes de satisfacción profesional que los hombres. Los empresarios varones preferían los satisfactores asociados con el logro del estatus (por ejemplo, ganar mucho dinero, tener un alto prestigio y estatus social, estar en un papel de liderazgo, liderar una empresa grande y de rápido crecimiento), mientras que las empresarias preferían los satisfactores asociados con las relaciones con los empleados (por ejemplo, trabajar con personas amigables y agradables, tener empleados de apoyo, trabajar como parte de un equipo) y hacer una contribución a la sociedad. Sin embargo, el estudio no presenta evidencia sobre si la masculinidad de los dueños de negocios se relaciona más fuertemente con las intenciones de crecimiento en comparación con la feminidad de los dueños de negocios.

\section{Condiciones de Contorno: El rol of Autoconstrucción Independiente}

La investigación proporciona evidencia convincente de que el concepto de actividad empresarial está sesgado por el género: el espíritu empresarial a menudo se representa como una forma de masculinidad y los términos "empresario" y "hombre" han tendido a ser intercambiables (Ahl y Marlow 2012; Gupta et al., 2009). Esto apoya una valoración jerárquica en la que se prioriza lo masculino sobre lo femenino y las características de los dueños de negocios exitosos (es decir, proactividad, necesidad de logro, competitividad en la toma de riesgos, confianza) se perciben estereotípicamente como masculinas (Ahl, 2006; Pájaro y pincel, 2002; Coleman, 2016). Además, el comportamiento de las mujeres involucradas en la actividad empresarial se define y evalúa de acuerdo con los estándares de una norma masculina invisible (Lewis, 2006). Las mujeres empresarias tienen que gestionar diferentes identidades simultáneamente, lo que puede dar lugar a conflictos (Ahl, 2006; Pájaro y pincel, 2002).

En consecuencia, para ser reconocidas como actores creíbles dentro del emprendimiento, las mujeres tienen que aprender el delicado equilibrio entre adoptar una identidad empresarial creíble que refleje normas 
masculinizadas pero sin negar la feminidad atribuida (Ahl \& Marlow 2012). Por lo tanto, una pregunta importante planteada es cómo se forman las intenciones de crecimiento de las mujeres empresarias a pesar de los mensajes mixtos que reciben de un proceso que enfatiza ampliamente la masculinidad, pero también espera que las mujeres cuiden y cuiden específicamente a los niños o mantengan el hogar, o que sean solidarias, es decir, interdependientes y conectadas con los demás. Sobre la base de las dimensiones culturales del individualismo y el colectivismo, Cross y Madson (1997) proponen facetas adicionales de la identidad de género que reflejan la inversión en un individuo frente al sentido social de sí mismo. Los autores definen la identidad masculina como un sentido independiente del yo y la identidad femenina como un sentido interdependiente del yo.

Específicamente, las autointerpretaciones independientes e interdependientes representan uno de los esquemas más fundamentales y generales de la identidad de un individuo (Markus y Kitayama, 1991; Oyserman et al., 2002). La autoconstrucción se refiere a la forma en que un individuo se entiende a sí mismo en relación con otras personas. Una persona que posee una autoconstrucción independiente se ve a sí misma como separada de los demás, con el objetivo de mantener un sentido de autonomía y singularidad. Una persona que posee una autoconstrucción interdependiente ve su yo como conectado con los demás, donde las auto-presentaciones se entrelazan con representaciones de otros cercanos y contexto social (Markus \& Kitayama, 1991; Oyserman et al., 2002). Es más probable que los hombres sean socializados para construir una autoconstrucción independiente y desarrollar un yo social que se caracterice por poner énfasis primario en los valores de las necesidades individuales, la autonomía y la autorrealización. Las mujeres son más propensas a ser socializadas para construir una autoconstrucción interdependiente y desarrollar un yo social que está marcado por la motivación de estar conectadas con relaciones o contextos particulares (Cross \& Madson, 1997; Oyserman et al., 2002; Singelis, 1994).

Hay investigaciones limitadas hasta la fecha que han evaluado la relación entre la autoconstrucción y el espíritu empresarial. Investigaciones empíricas recientes proporcionan evidencia del papel moderador de la autoconstrucción a nivel individual en los modelos cognitivos de intención de iniciar un nuevo negocio (Siu \& Lo, 2013; Zampetakis et al., 2015). Por ejemplo, Siu y Lo (2013), utilizando una muestra de estudiantes de China y Hong Kong, encontraron que la fuerza de las normas sociales percibidas para predecir la intención empresarial dependía de la autoconstrucción interdependiente. Zampetakis et al. (2015) utilizando una muestra de estudiantes de Grecia encontraron que la autoconstrucción independiente estaba relacionada con las actitudes hacia el emprendimiento y las relaciones moderadas entre las actitudes y las intenciones empresariales.

Como se señaló anteriormente, para que las mujeres ganen legitimidad como empresarias, se les alienta a adoptar y reproducir actitudes y comportamientos que de hecho son reproducciones de lo que hacen los hombres y lo que son los hombres (Ahl y Marlow, 2012). Sin embargo, algunas pruebas sugieren que cuando las mujeres emulan el comportamiento del hombre idealizado, hay evidencia de inadaptación. Por ejemplo, Kerfoot y Miller (2010) evaluaron los resultados de un programa de capacitación para posibles dueños de negocios con el objetivo de alentar a más mujeres a iniciar sus propias empresas. El programa estableció a los hombres como empresarios naturales que convierten a las mujeres en forasteras, y la única esperanza para ingresar en el proceso empresarial era aprender a emular el comportamiento del hombre idealizado. Después de emprender el curso, muchas de las posibles propietarias de negocios se desalentaron activamente de comenzar nuevas empresas. Se hizo evidente que no "encajaban" en la imagen masculinizada de un emprendedor (Kerfoot \& Miller, 2010).

Esto sugiere que cuando las señales o símbolos en el entorno hacen que la autoconstrucción independiente sea más accesible (Hong et al., 2000; Oyserman y Sorensen, 2009) (es decir, símbolos de libertad individual, autonomía, realización personal y separación), entonces las empresarias femeninas pueden tener menos necesidad de hacer crecer sus empresas debido al conflicto inherente a la relación entre la autoconstrucción independiente y la feminidad. Específicamente, las personas que se ven a sí mismas como más altas en feminidad son más propensas a considerarse a sí mismas como miembros de un grupo. Esto, a su vez, provoca un estilo cognitivo que se preocupa por las consecuencias negativas del comportamiento (es decir, el crecimiento del negocio) y evitar el fracaso potencial (Oyserman y Sorensen, 
2009). Por lo tanto, esperamos que la interacción entre la autoconstrucción independiente y la feminidad para la predicción del crecimiento sea negativa.

\section{Visión General of Estudio Objetivos de Unae Hipótesis}

El propósito del presente estudio fue examinar las diferencias en el proceso de crecimiento empresarial de las empresas establecidas, entre empresarios y empresarias. Nos centramos en un aspecto específico del crecimiento de la empresa, la intención del empresario, y si y cómo el sexo biológico, la identidad de género y la autointerpretación independiente afectan las intenciones de crecimiento.

Específicamente, el estudio tuvo como objetivo determinar en primer lugar si y en qué medida el sexo biológico está asociado con la identidad de género y las intenciones de crecimiento empresarial de los empresarios. Esperábamos que la correlación entre el sexo de los empresarios y la identidad de género fuera fuerte, de modo que los empresarios masculinos fueran más propensos a exhibir una orientación masculina estereotipada y las empresarias más propensas a exhibir una orientación femenina estereotipada. En segundo lugar, nuestro objetivo fue probar si, y en qué medida, la identidad de género media los efectos biológicos del sexo en las intenciones de crecimiento empresarial. Esperábamos que la identidad de género mediaría al menos parcialmente los efectos biológicos del sexo en las intenciones de crecimiento; esperábamos que el efecto de la masculinidad sobre las intenciones de crecimiento fuera más fuerte en comparación con el efecto de la feminidad. Finalmente, examinamos las influencias moderadoras de la autointerpretación independiente que constituyen las condiciones de contorno de la teoría. Esperábamos que la autointerpretación independiente moderara la relación entre el sexo y la identidad de género y entre la identidad de género y las intenciones de crecimiento. Hasta donde sabemos, el presente estudio es uno de los primeros en examinar con cierta profundidad las orientaciones culturales a nivel individual dentro de la cultura como antecedentes de las intenciones de crecimiento empresarial.

Realizamos un análisis de mediación moderado (Hayes, 2013) con el fin de distinguir claramente el poder mediador de la identidad de género y el papel moderador de la autoconstrucción independiente mientras se controlan las variables tanto a nivel de empresa como a nivel individual.

\section{CONCLUSIÓN}

Los hallazgos del presente estudio dejan en claro que la intención de crecimiento empresarial es un fenómeno complejo que puede estar influenciado por el género. Nuestros resultados indican que la identidad de género media la influencia del sexo en las intenciones de crecimiento empresarial y los efectos de la mediación dependen de la autointerpretación independiente de los empresarios. Por lo tanto, las mujeres toman decisiones relacionadas con el crecimiento de sus negocios utilizando un proceso diferente al de los hombres. Nuestros resultados refuerzan las afirmaciones de que es valioso incorporar una perspectiva femenina al estudiar los factores que influyen en las intenciones de crecimiento de los empresarios en los negocios establecidos.

\section{TRANSLATED VERSION: FRENCH}

Below is a rough translation of the insights presented above. This was done to give a general understanding of the ideas presented in the paper. Please excuse any grammatical mistakes and do not hold the original authors responsible for these mistakes.

\section{VERSION TRADUITE: FRANÇAIS}

Voici une traduction approximative des idées présentées ci-dessus. Cela a été fait pour donner une compréhension générale des idées présentées dans le document. Veuillez excuser toutes les erreurs grammaticales et ne pas tenir les auteurs originaux responsables de ces erreurs. 


\section{INTRODUCTION}

Le développement et la croissance des entreprises existantes est un processus qui présente de nombreux avantages sociétaux, notamment la création d'emplois et de richesses et l'avancement de l'innovation (Tang et Koveos, 2004; Van Praag et Versloot, 2007). La décision des entrepreneurs de faire croître leur entreprise est complexe, n'est ni linéaire ni dépendante d'un nombre limité de facteurs (Miller et coll., 2013). Les entrepreneurs doivent non seulement prendre des décisions importantes pour différentes tâches de nature différente, mais aussi s'assurer que ces décisions sont les bonnes non seulement pour leur entreprise, mais aussi pour eux-mêmes. Alors que certains entrepreneurs sont heureux d'être des travailleurs autonomes à petite échelle, d'autres ont des aspirations de croissance pour leurs entreprises (Shane, 2009).

La recherche montre clairement que les entreprises appartenant à des femmes ont tendance à avoir des niveaux de croissance plus faibles et demeurent plus petites que les entreprises appartenant à des hommes (Cliff, 1998; Coleman, 2016; Davis \& Shaver, 2012), suggérant l'existence d'un écart entre les hommes et les femmes dans l'entrepreneuriat; il s'agit d'une question qui attire de plus en plus l'attention des universitaires (Coleman, 2016). Cependant, une grande partie de cette recherche a étudié les différences dans les niveaux d'activité de démarrage entre les hommes et les femmes (voir Jennings \& Brush, 2013). Peu de recherches ont examiné les aspirations de croissance des entreprises dirigées par des femmes. Comparativement aux hommes entrepreneurs, les femmes entrepreneurs ont tendance à poursuivre des objectifs non économiques tels que l'équilibre entre le travail et les rôles familiaux et ont des préférences pour les relations avec les employés et les satisfaisants de la société, ce qui peut à son tour nuire à la performance ou à la croissance économiques (Eddleston et Powell, 2008; Jennings et Brush, 2013).

Comme l'ont noté Henry et al. (2016), les représentations normatives de l'entrepreneuriat sont dominées par la masculinité. Cela marginalise les femmes entrepreneures et les rend invisibles. Les auteurs notent que si la prise de conscience que le genre (masculin et féminin) est socialement construit et correspond à un ensemble appris de comportements qui ne se réfère pas simplement au sexe biologique (homme / homme et femme / femme) de l'entrepreneur semble répandue parmi les chercheurs, plusieurs thèmes préjudiciables liés aux femmes sont persistants. Par exemple, de nombreux chercheurs utilisent des normes masculines pour juger les activités des femmes en comparant simplement les hommes et les femmes, avec peu ou pas d'attention accordée aux constructions du genre (Ahl, 2006; Lewis, 2006). Cependant, cette pratique néglige le fait que les aspects masculins et féminins seront intégrés dans le processus de croissance des entreprises pour les hommes et les femmes. C'est-à-dire que le genre pourrait être un aspect vital de l'intention de croissance de l'entreprise.

Dans l'ensemble, à ce jour, la recherche ne fournit pas d'explications concluantes à la variation de la trajectoire de croissance que les hommes et les femmes aimeraient que leur entreprise suive (c.-à-d. leur intention de croissance) (Bulanova et coll., 2016; Dutta et Thornhill, 2008). L'intention de croissance est considérée comme une caractéristique essentielle du comportement entrepreneurial et un élément clé dans la compréhension du développement et de la croissance des entreprises (Sadler-Smith et al. 2003). De plus, les intentions se sont avérées être le meilleur prédicteur du comportement planifié (Ajzen, 1991), en particulier si le phénomène implique des décalages temporels imprévisibles, une planification et un degré élevé de traitement cognitif, comme la croissance des entreprises (Krueger et al. 2000). Les intentions de croissance sont considérées comme un prédicteur clé de la croissance réelle des activités (Delmar et Wiklund, 2008; Wiklund et Shepherd, 2003).

De nombreuses preuves suggèrent que l'identité (ou le concept de soi) d'un individu en général (Fauchart et Gruber, 2011; Hoang et Gimeno, 2010) et l'identité de genre (ou les rôles de genre connexes) en particulier (Eddleston et Powell, 2008; Gupta et al., 2009) peuvent contribuer à des différences substantielles entre les hommes et les femmes dans le comportement entrepreneurial. Les gens ont tendance à différer dans la mesure où ils intègrent les rôles de genre dans leurs concepts de soi.

Dans la présente étude, nous proposons que l'identité de genre des entrepreneurs ou la mesure dans laquelle les entrepreneurs possèdent des traits associés aux stéréotypes de genre traditionnels (Bem's Sex

Role Inventory, 1981; Mueller, 2004; Wood \& Eagly, 2010) est un mécanisme cognitif important qui relie le sexe des entrepreneurs aux intentions de croissance de l'entreprise. Nous soutenons que l'identité de 
genre façonne la façon dont les entrepreneurs se voient, comment ils comprennent le monde qui les entoure et abordent les autres, mais aussi ce qu'ils visent à réaliser à l'avenir (Eddleston et Powell, 2008; Hoang et Gimeno, 2010).

Cependant, les mécanismes par lesquels le sexe et l'identité de genre exercent une influence sur les intentions de croissance des entrepreneurs et les influences modérateurs qui constituent les conditions limites de la théorie doivent faire l'objet d'une enquête plus approfondie. L'auto-interprétation est un modérateur potentiel de la relation entre le genre, l'identité de genre et les intentions de croissance. L'autointerprétation fait référence aux pensées, aux sentiments et aux actions culturellement contingents des individus qui sont concernés par la compréhension de soi comme étant connecté aux autres (autointerprétation interdépendante) ou distinct des autres (auto-interprétation indépendante) (Markus et Kitayama, 1991). L'exposition des individus à certaines affections peut accroître la probabilité qu'ils présentent des tendances indépendantes (individualistes) ou interdépendantes (collectivistes) (Hong et coll., 2000). Les concepts d'« entrepreneur » et d'« entrepreneuriat» sont considérés comme masculins (Ahl, 2006; Lewis, 2006). Les indices ou les symboles dans l'environnement que les entrepreneurs utilisent pour exploiter leur entreprise pourraient rendre l'auto-interprétation indépendante plus accessible (Hong et al., 2000), puisque l'identité masculine met en évidence les attributs uniques des individus (Cross et Madson, 1997). Un examen systématique des effets modérateurs potentiels de l'auto-interprétation contribuera à une meilleure compréhension des conditions dans lesquelles les effets du sexe sur les intentions de croissance sont plus susceptibles de se maintenir.

En résumé, l'objectif de cet article est d'approfondir la compréhension des facteurs qui influencent la motivation de croissance des entreprises dans les entreprises établies. À cette fin, l'un des principaux objectifs de la présente étude est d'examiner la relation entre le sexe, l'identité de genre et les intentions de croissance des propriétaires d'entreprise de leurs entreprises établies. Nous avons estimé qu'une exploration des intentions de croissance des propriétaires d'entreprises établies pourrait aider à expliquer les différences entre les sexes dans la décision de croître. Un deuxième objectif majeur de la présente étude est de se concentrer sur l'auto-interprétation des entrepreneurs en tant que modérateur potentiel de la relation entre le sexe et l'identité de genre et entre l'identité de genre et l'intention de croissance de l'entreprise (voir le modèle conceptuel à la figure 1). À notre connaissance, à ce jour, il y a eu peu de recherches expliquant l'influence de ces domaines et l'intention des entrepreneurs pour la croissance de l'entreprise établie. Cependant, cela est important étant donné que les individus démarrent et exploitent leurs entreprises pour diverses raisons autres que la croissance ou la maximisation des rendements économiques (Wiklund et al. 2003).

Le document commence par une analyse des intentions de croissance des entreprises; elle est suivie d'une évaluation de l'influence de l'identité de genre sur les intentions de croissance et du rôle modérateur de l'auto-interprétation. Ensuite, nous décrivons notre échantillon, nos méthodes de recherche et nos techniques d'analyse de données, notamment des analyses aNOVA et des analyses de médiation modérées. Enfin, nous discutons des résultats de l'analyse empirique et des limites de la recherche, présentons l'implication pratique des résultats de la recherche et proposons des domaines de recherche supplémentaires.

\section{Intentionde Croissance et Identité de Genredes Entrepreneurs}

La croissance de l'entreprise est un phénomène multidimensionnel et complexe qui implique une planification et une réflexion minutieuses de la part de l'entrepreneur. En tant que telle, la croissance des entreprises peut être considérée comme un comportement intentionnel délibéré et planifié et, par conséquent, applicable aux modèles d'intention (Ajzen, 1991; Krueger et coll., 2000). Les intentions de croissance sont au cœur de la compréhension de la croissance des entreprises, car elles constituent la première étape du processus et impliquent un élément objectif avec des étapes spécifiques pour atteindre l'objectif (Delmar et Wiklund, 2008; Venugopal, 2016). Les intentions ont été identifiées comme un prédicteur clé du comportement réel, à travers un large éventail de comportements différents, y compris le démarrage d'entreprise (Delmar et Wiklund, 2008; Wiklund et Shepherd, 2003). De plus, la recherche fournit des preuves que la proportion d'entrepreneurs ayant des intentions de croissance dans la population 
est un prédicteur important de la croissance économique par rapport aux taux de travail autonome ou aux taux généraux de démarrage (Stam et coll., 2009).

À ce jour, la recherche sur les intentions de croissance des entrepreneurs a donné des résultats mitigés en ce qui concerne les différences empiriques entre les hommes et les femmes, et tous les chercheurs ne s'entendent pas pour dire que les femmes et les hommes diffèrent dans leur intention globale de croissance des entreprises (Cassar, 2006; Davis et Shaver, 2012). Par exemple, Cassar (2006) a constaté que les femmes entrepreneures avaient des estimations de revenus futurs inférieures à celles des hommes. Cependant, Menzies et coll. (2004), dans une étude menée au Canada, n'ont trouvé aucune différence statistiquement significative entre les femmes et les hommes dans leurs préférences exprimées pour une croissance effrénée. Dans la présente étude, nous proposons que les processus de socialisation de genre peuvent expliquer les différences entre les intentions de croissance des entreprises des hommes et des femmes entrepreneurs.

La recherche contemporaine en entrepreneuriat suggère que des décisions telles que la croissance d'entreprises établies sont intimement liées à l'identité des entrepreneurs (Shepherd \& Haynie 2009). L'identité est un processus social fluide qui a une gamme de significations conceptuelles et de rôles théoriques qui lui sont associés (Fauchart \& Gruber, 2011; Hoang et Gimeno, 2010). Il peut être considéré comme la représentation par les gens de l'intériorisation et de l'incorporation des attentes comportementales socialement détenues. De plus, l'identité peut être considérée comme un concept fondamental de pont entre l'individu et le social (Hoang \& Gimeno, 2010). Les individus sont socialement construits à travers des interactions sociales et qu'ils acquièrent tout au long de leur vie des identités sociales diverses et multiples. Dans le cas du processus entrepreneurial, Ireland et Webb (2007) notent dans leur revue que pour les entrepreneurs, le processus entrepreneurial est basé sur l'identité de soi et motivé par celle-ci. Les modèles conceptuels récents du rôle de l'identité dans l'entrepreneuriat proposent des liens étroits entre le concept de soi des entrepreneurs et les actions et les résultats entrepreneuriaux (Shepherd \& Haynie 2009), mais à ce jour, la recherche empirique est limitée (Farmer et al. 2011). L'identité de genre est considérée comme un élément essentiel d'une conception multidimensionnelle du soi qui situe les individus au sein des structures sociales (Eddleston et Powell, 2008; Wood et Eagly, 2010). Le genre est différent du sexe (Ahl, 2006). Les chercheurs ont distingué le sexe du genre au cours des années 1970 et 1980 afin de faire la distinction entre les caractéristiques biologiques des hommes et des femmes et les significations que les sociétés et les individus attribuent aux catégories masculines et féminines (Bem's Sex Role Inventory, 1981; Wood et Eagly, 2010). Tout au long de la vie, on enseigne (implicitement ou explicitement) aux individus quels comportements et rôles sont souhaitables pour les hommes ou les femmes dans la société. En tant que tels, la plupart des individus acceptent ou intériorisent les significations culturelles associées à leurs significations sexuelles. L'identité de genre représente la mesure dans laquelle une personne croit qu'elle possède des traits associés aux stéréotypes de genre traditionnels (Bem's Sex Role Inventory, 1981; Wood et Eagly, 2010).

Les gens diffèrent dans la mesure où ils intègrent les rôles de genre dans leurs concepts de soi. Deux dimensions indépendantes de l'identité de genre se posent : la masculinité, ou les croyances sur la mesure dans laquelle on possède des traits associés aux hommes (p. ex., la domination, l'indépendance, l'ambition) et la féminité, ou les croyances sur la mesure dans laquelle on possède des traits associés aux femmes (p. ex., sensibilité aux besoins des autres, compassion, compréhension) (Eddleston et Powell, 2008; Wood et Eagly, 2010). Les femmes sont socialisées pour s'identifier à des attributs considérés comme féminins, et les hommes sont socialisés pour s'identifier à des attributs considérés comme masculins (Bem's Sex Role Inventory, 1981). Ainsi, les hommes entrepreneurs sont plus susceptibles de présenter une orientation masculine stéréotypée et les femmes entrepreneurs sont plus susceptibles d'afficher une orientation féminine stéréotypée (Bird \& Brush, 2002).

Les gens utilisent leur identité de genre comme une norme contre laquelle réglementer leur comportement. Les entrepreneurs qui ont un concept de soi masculin impliquant l'indépendance et la domination peuvent réglementer leur comportement, par exemple, en recherchant des opportunités de croissance élevée et des récompenses financières de leurs entreprises (Bird \& Brush, 2002). Eddleston et Powell (2008), dans leur enquête auprès des anciens entrepreneurs, montrent que les femmes entrepreneures 
valorisent des sources de satisfaction professionnelle différentes de celles des hommes. Les entrepreneurs de sexe masculin préféraient les personnes satisfaites associées à l'obtention d'un statut (p. ex., gagner beaucoup d'argent, avoir un prestige et un statut social élevés, être dans un rôle de leadership, diriger une grande entreprise en croissance rapide), tandis que les femmes entrepreneures préféraient les femmes qui étaient satisfaites associées aux relations avec les employés (p. ex., travailler avec des personnes amicales et agréables, avoir des employés qui soutiennent, travailler en équipe) et apporter une contribution à la société. Néanmoins, l'étude ne présente aucune preuve quant à savoir si la masculinité des propriétaires d'entreprise est plus fortement liée aux intentions de croissance qu'à la féminité des propriétaires d'entreprise.

\section{Conditions Aux Limites : Le Rôle Del'auto-Interprétation Indépendante}

La recherche fournit des preuves convaincantes que le concept d'activité entrepreneuriale est sexiste : l'entrepreneuriat est souvent décrit comme une forme de masculinité et les termes « entrepreneur » et " homme » ont eu tendance à devenir interchangeables (Ahl \& Marlow 2012; Gupta et coll., 2009). Cela appuie une évaluation hiérarchique dans laquelle le masculin est prioritaire sur le féminin et les caractéristiques des propriétaires d'entreprise qui réussissent (c.-à-d. la proactivité, le besoin de réussite, la compétitivité de la prise de risque, la confiance) sont stéréotypées comme faisant l'objet d'un masculin (Ahl, 2006; Bird \& Brush, 2002; Coleman, 2016). De plus, le comportement des femmes impliquées dans l'activité entrepreneuriale est défini et évalué selon les normes d'une norme masculine invisible (Lewis, 2006). Les femmes entrepreneures doivent gérer simultanément différentes identités, ce qui peut entraîner des conflits (Ahl, 2006; Bird \& Brush, 2002).

En conséquence, pour être reconnues comme des actrices crédibles au sein de l'entrepreneuriat, les femmes doivent apprendre l'équilibre délicat entre l'adoption d'une identité entrepreneuriale crédible qui reflète des normes masculinisées mais sans nier la féminité attribuée (Ahl \& Marlow 2012). Ainsi, une question importante soulevée est de savoir comment les intentions de croissance des femmes entrepreneures se forment malgré les messages contradictoires qu'elles reçoivent d'un processus qui met largement l'accent sur la masculinité, mais qui s'attend également à ce que les femmes s'occupent spécifiquement des enfants et les nourrissent ou maintiennent le ménage, ou soient solidaires, c'est-à-dire interdépendantes et connectées aux autres. S'appuyant sur les dimensions culturelles de l'individualisme et du collectivisme, Cross et Madson (1997) proposent d'autres facettes de l'identité de genre reflétant l'investissement dans un sentiment individuel par rapport au sens social de soi. Les auteurs définissent l'identité masculine comme un sens indépendant de soi et l'identité féminine comme un sens interdépendant de soi.

Plus précisément, les auto-interprétations indépendantes et interdépendantes représentent l'un des schémas les plus fondamentaux et les plus globaux de l'égoïsme d'un individu (Markus et Kitayama, 1991; Oyserman et coll., 2002). L'auto-interprétation fait référence à la façon dont un individu se comprend par rapport aux autres. Une personne possédant une auto-interprétation indépendante se considère comme séparée des autres, dans le but de maintenir un sentiment d'autonomie et d'unicité. Une personne possédant une auto-interprétation interdépendante considère son soi comme connecté aux autres, où les présentations de soi sont tissées ensemble avec des représentations d'autres proches et du contexte social (Markus et Kitayama, 1991; Oyserman et coll., 2002). Les hommes sont plus susceptibles d'être socialisés pour construire une auto-interprétation indépendante et développer un soi social qui est marqué par l'accent mis en mettant l'accent sur les valeurs des besoins individuels, de l'autonomie et de l'épanouissement personnel. Les femmes sont plus susceptibles d'être socialisées pour construire une auto-interprétation interdépendante et développer un soi social qui est marqué par la motivation d'être connecté à des relations ou des contextes particuliers (Cross \& Madson, 1997; Oyserman et coll., 2002; Singelis, 1994).

À ce jour, peu de recherches ont évalué la relation entre l'auto-interprétation et l'entrepreneuriat. Des recherches empiriques récentes fournissent des preuves du rôle modérateur de l'auto-interprétation au niveau individuel dans les modèles cognitifs d'intention de démarrer une nouvelle entreprise (Siu \& Lo, 2013; Zampetakis et coll., 2015). Par exemple, Siu et Lo (2013), en utilisant un échantillon d'étudiants de Chine et de Hong Kong, ont constaté que la force des normes sociales perçues dans la prédiction de l'intention entrepreneuriale dépendait de l'auto-interprétation interdépendante. Zampetakis et al. (2015) en 
utilisant un échantillon d'étudiants grecs ont constaté que l'auto-interprétation indépendante était liée aux attitudes envers l'entrepreneuriat et aux relations modérées entre les attitudes et les intentions entrepreneuriales.

Comme indiqué ci-dessus, pour que les femmes acquièrent une légitimité en tant qu'entrepreneures, elles sont encouragées à adopter et à reproduire des attitudes et des comportements qui sont en fait des reproductions de ce que font les hommes et de ce que sont les hommes (Ahl \& Marlow, 2012). Cependant, certaines preuves suggèrent que lorsque les femmes imitent le comportement de l'homme idéalisé, il existe des preuves d'inadéquation. Par exemple, Kerfoot et Miller (2010) ont évalué les résultats d'un programme de formation destiné aux propriétaires d'entreprise potentiels visant à encourager un plus grand nombre de femmes à créer leur propre entreprise. Le programme a établi les hommes comme des entrepreneurs naturels faisant des femmes des outsiders, et le seul espoir d'entrer dans le processus entrepreneurial était d'apprendre à imiter le comportement de l'homme idéalisé. Après avoir suivi le cours, bon nombre des femmes propriétaires d'entreprise potentielles ont été activement découragées de démarrer de nouvelles entreprises. Il est devenu évident qu'ils ne « correspondaient » pas à l'image masculinisée d'un entrepreneur (Kerfoot \& Miller, 2010).

Cela donne à penser que lorsque des indices ou des symboles dans l'environnement rendent l'autointerprétation indépendante plus accessible (Hong et coll., 2000; Oyserman \& Sorensen, 2009) (c'est-à-dire symboles de liberté individuelle, d'autonomie, d'épanouissement personnel et de séparation), les femmes entrepreneurs peuvent avoir moins besoin de développer leurs entreprises en raison du conflit inhérent à la relation entre l'auto-interprétation indépendante et la féminité. Plus précisément, les personnes qui se considèrent comme plus élevées dans la féminité sont plus susceptibles de se considérer comme un membre d'un groupe. Cela incite à son tour à un style cognitif qui se préoccupe des conséquences négatives du comportement (c'est-à-dire la croissance des entreprises) et évite l'échec potentiel (Oyserman \& Sorensen, 2009). Ainsi, nous nous attendons à ce que l'interaction entre l'auto-interprétation indépendante et la féminité pour la prédiction de la croissance soit négative.

\section{Aperçu Desobjectifs De L'étudeet Hypothèses}

L'objectif de la présente étude était d'examiner les différences dans le processus de croissance des entreprises établies, entre les hommes et les femmes entrepreneurs. Nous nous sommes concentrés sur un aspect spécifique de la croissance de l'entreprise - l'intention de l'entrepreneur - et sur la question de savoir si et comment le sexe biologique, l'identité de genre et l'auto-interprétation indépendante affectent les intentions de croissance.

Plus précisément, l'étude visait tout d'abord à déterminer si et dans quelle mesure le sexe biologique est associé à l'identité de genre et aux intentions de croissance des entreprises des entrepreneurs. Nous nous attendions à ce que la corrélation entre le sexe et l'identité de genre des entrepreneurs soit forte, de sorte que les entrepreneurs masculins sont plus susceptibles de présenter une orientation masculine stéréotypée et que les femmes entrepreneurs sont plus susceptibles de présenter une orientation féminine stéréotypée. Deuxièmement, nous avons cherché à vérifier si, et dans quelle mesure, l'identité de genre intéresse les effets biologiques du sexe sur les intentions de croissance des entreprises. Nous nous attendions à ce que l'identité de genre médie au moins partiellement les effets biologiques du sexe sur les intentions de croissance; nous nous attendions à ce que l'effet de la masculinité sur les intentions de croissance soit plus fort que l'effet de la féminité. Enfin, nous avons examiné les influences modérateurs de l'auto-interprétation indépendante qui constituent les conditions limites de la théorie. Nous nous attendions à ce que l'autointerprétation indépendante modère la relation entre le sexe et l'identité de genre et entre l'identité de genre et les intentions de croissance. À notre connaissance, la présente étude est l'une des premières à examiner en profondeur les orientations culturelles au niveau individuel au sein de la culture en tant qu'antécédents des intentions de croissance des entreprises.

Nous avons mené une analyse modérée de la médiation (Hayes, 2013) afin de distinguer clairement le pouvoir de médiation de l'identité de genre et le rôle modérateur de l'auto-interprétation indépendante tout en contrôlant les variables au niveau de l'entreprise et au niveau individuel. 


\section{CONCLUSION}

Les résultats de la présente étude montrent clairement que l'intention de croissance des entreprises est un phénomène complexe qui peut être influencé par le sexe. Nos résultats indiquent que l'identité de genre sert de médiateur dans l'influence du sexe sur les intentions de croissance des entreprises et que les effets de la médiation dépendent de l'auto-interprétation indépendante des entrepreneurs. Ainsi, les femmes prennent des décisions liées à la croissance de leur entreprise en utilisant un processus différent de celui des hommes. Nos résultats renforcent les affirmations selon lesquelles il est utile d'intégrer une perspective féminine lors de l'étude des facteurs influençant les intentions de croissance des entrepreneurs dans les entreprises établies.

\section{TRANSLATED VERSION: GERMAN}

Below is a rough translation of the insights presented above. This was done to give a general understanding of the ideas presented in the paper. Please excuse any grammatical mistakes and do not hold the original authors responsible for these mistakes.

\section{ÜBERSETZTE VERSION: DEUTSCH}

Hier ist eine ungefähre Übersetzung der oben vorgestellten Ideen. Dies wurde getan, um ein allgemeines Verständnis der in dem Dokument vorgestellten Ideen zu vermitteln. Bitte entschuldigen Sie alle grammatikalischen Fehler und machen Sie die ursprünglichen Autoren nicht für diese Fehler verantwortlich.

\section{EINLEITUNG}

Die Entwicklung und das Wachstum bestehender Unternehmen ist ein Prozess mit vielen gesellschaftlichen Vorteilen, einschließlich der Schaffung von Arbeitsplätzen und Wohlstand und der Förderung von Innovationen (Tang \& Koveos, 2004; Van Praag \& Versloot, 2007). Die Entscheidung von Unternehmern, ihr Geschäft auszubauen, ist komplex, weder linear noch abhängig von einer begrenzten Anzahl von Faktoren (Miller et al. 2013). Unternehmer müssen nicht nur wichtige Entscheidungen für verschiedene Aufgaben treffen, die unterschiedlicher Natur sind, sondern auch sicherstellen, dass diese Entscheidungen nicht nur für ihr Unternehmen, sondern auch für sich selbst die richtigen sind. Während einige Unternehmer froh sind, in kleinem Maßstab selbstständig zu sein, haben andere Wachstumsziele für ihre Unternehmungen (Shane, 2009).

Die Forschung zeigt deutlich, dass Unternehmen in Frauenbesitz tendenziell ein geringeres Wachstum aufweisen und kleiner bleiben als Unternehmen in Männerbesitz (Cliff, 1998; Coleman, 2016; Davis \& Shaver, 2012), die auf die Existenz einer Kluft zwischen Männern und Frauen im Unternehmertum hindeutet; Dies ist ein Thema, das zunehmend akademische Aufmerksamkeit erregt (Coleman, 2016). Ein Großteil dieser Forschung hat jedoch Unterschiede in der Start-up-Aktivität zwischen Männern und Frauen untersucht (siehe Jennings \& Brush, 2013). Wenig Forschung hat die Wachstumsbestrebungen von Unternehmen untersucht, die von Frauen geleitet werden. Im Vergleich zu männlichen Unternehmern neigen Unternehmerinnen dazu, nichtwirtschaftliche Ziele wie die Vereinbarkeit von Arbeit und Familie zu verfolgen und haben Präferenzen für Mitarbeiterbeziehungen und gesellschaftliche Befriedigungsfaktoren, die wiederum die Wirtschaftsleistung oder das Wachstum beeinträchtigen können (Eddleston \& Powell, 2008; Jennings \& Brush, 2013).

Wie Henry et al. (2016) feststellten, werden normative Repräsentationen von Unternehmertum von Männlichkeit dominiert. Das marginalisiert Unternehmerinnen und macht sie unsichtbar. Die Autoren stellen fest, dass, während das Bewusstsein, dass Geschlecht (männlich und weiblich) sozial konstruiert ist und einer erlernten Reihe von Verhaltensweisen entspricht, die sich nicht nur auf das biologische Geschlecht (männlich / Mann und weiblich / Frau) des Unternehmers beziehen, unter Wissenschaftlern weit 
verbreitet zu sein scheint, mehrere schädliche Themen im Zusammenhang mit Frauen hartnäckig sind. Zum Beispiel verwenden viele Wissenschaftler männliche Normen, um die Aktivitäten von Frauen zu beurteilen, indem sie nur Männer und Frauen vergleichen, wobei den Konstruktionen des Geschlechts wenig oder gar keine Aufmerksamkeit geschenkt wird (Ahl, 2006; Lewis, 2006). Diese Praxis vernachlässigt jedoch die Tatsache, dass männliche und weibliche Aspekte in den Prozess des Geschäftswachstums für Männer und Frauen einbezogen werden. Das heißt, das Geschlecht könnte ein wichtiger Aspekt der Wachstumsabsicht des Unternehmens sein.

Zusammengenommen liefert die Forschung bisher keine schlüssigen Erklärungen für die Variation des Wachstumspfads, die Männer und Frauen ihrem Unternehmen folgen möchten (dh ihrer Wachstumsabsicht) (Bulanova et al. 2016; Dutta \& Thornhill, 2008). Wachstumsabsicht gilt als wesentliches Merkmal unternehmerischen Verhaltens und als Schlüsselelement für das Verständnis von Venture Development und Growth (Sadler-Smith et al. 2003). Darüber hinaus haben sich Absichten als der beste Prädiktor für geplantes Verhalten erwiesen (Ajzen, 1991), insbesondere wenn das Phänomen unvorhersehbare Zeitverzögerungen, Planung und ein hohes Maß an kognitiver Verarbeitung wie Geschäftswachstum beinhaltet (Krueger et al. 2000). Wachstumsabsichten gelten als wichtiger Prädiktor für das tatsächliche Geschäftswachstum (Delmar \& Wiklund, 2008; Wiklund \& Shepherd, 2003).

Beträchtliche Beweise deuten darauf hin, dass die Identität (oder das Konzept des Selbst) eines Individuums im Allgemeinen (Fauchart \& Gruber, 2011; Hoang \& Gimeno, 2010) und insbesondere Geschlechtsidentität (oder verwandte Geschlechterrollen) (Eddleston \& Powell, 2008; Gupta et al., 2009) können zu erheblichen Unterschieden zwischen Männern und Frauen im unternehmerischen Verhalten beitragen. Menschen neigen dazu, sich darin zu unterscheiden, inwieweit sie Geschlechterrollen in ihr Selbstverständnis integrieren.

In der vorliegenden Studie schlagen wir vor, dass die Geschlechtsidentität von Unternehmern oder das Ausmaß, in dem Unternehmer Merkmale besitzen, die mit traditionellen Geschlechterstereotypen verbunden sind (Bem's Sex Role Inventory, 1981; Mueller, 2004; Wood \& Eagly, 2010) ist ein wichtiger kognitiver Mechanismus, der das Geschlecht von Unternehmern mit den Wachstumsabsichten des Unternehmens in Verbindung setzt. Wir argumentieren, dass geschlechtsidentität die Art und Weise prägt, wie Unternehmer sich selbst sehen, wie sie die Welt um sich herum verstehen und auf andere Menschen zugehen, aber auch, was sie in Zukunft erreichen wollen (Eddleston \& Powell, 2008; Hoang \& Gimeno, 2010).

Die Mechanismen, mit denen Geschlecht und Geschlechtsidentität Einfluss auf die Wachstumsabsichten von Unternehmern ausüben, und die moderierenden Einflüsse, die Randbedingungen der Theorie darstellen, bedürfen jedoch weiterer Untersuchungen. Selbstkonstruktion ist ein solcher potenzieller Moderator der Beziehung zwischen Geschlecht, Geschlechtsidentität und Wachstumsabsichten. Selbstkonstruktion bezieht sich auf kulturell bedingte Gedanken, Gefühle und Handlungen von Individuen, die sich mit dem Verständnis des Selbst als mit anderen verbunden (interdependent self-construal) oder von anderen (independent self-construal) befassen (Markus \& Kitayama, 1991). Die Exposition von Individuen gegenüber bestimmten Bedingungen kann die Wahrscheinlichkeit erhöhen, dass sie unabhängige (individualistische) oder voneinander abhängige (kollektivistische) Tendenzen aufweisen (Hong et al., 2000). Die Begriffe "Unternehmer" und "Unternehmertum" gelten als männlich geschlechtlich (Ahl, 2006; Lewis, 2006). Hinweise oder Symbole in der Umgebung, die Unternehmer verwenden, um ihr Geschäft zu betreiben, könnten unabhängige Selbstkonstruktion zugänglicher machen (Hong et al., 2000), da die männliche Identität die einzigartigen Attribute des Einzelnen hervorhebt (Cross \& Madson, 1997). Eine systematische Betrachtung der möglichen moderierenden Auswirkungen der Selbstkonstruktion wird zu einem umfassenderen Verständnis der Bedingungen beitragen, unter denen die Auswirkungen von Sex auf Wachstumsabsichten eher anhalten.

Zusammenfassend ist das Ziel dieses Artikels, das Verständnis der Faktoren zu vertiefen, die die Wachstumsmotivation in etablierten Unternehmen beeinflussen. Zu diesem Zweck ist es ein Hauptziel der vorliegenden Studie, die Beziehung zwischen dem Geschlecht, der Geschlechtsidentität und den Wachstumsabsichten der Unternehmer ihrer etablierten Unternehmen zu untersuchen. Wir argumentierten, 
dass eine Untersuchung der Wachstumsabsichten von Geschäftsinhabern etablierter Unternehmen dazu beitragen könnte, Geschlechtsunterschiede bei der Wachstumsentscheidung zu erklären. Ein zweiter Hauptzweck der vorliegenden Studie ist es, sich auf die Selbstkonstruktion von Unternehmern als potenzieller Moderator der Beziehung zwischen Geschlecht und Geschlechtsidentität sowie zwischen Geschlechtsidentität und Geschäftswachstumsabsicht zu konzentrieren (siehe konzeptionelles Modell in Abb. 1). Nach unserem besten Wissen gibt es bisher wenig Forschung, die den Einfluss dieser Domänen und die Absicht der Unternehmer für das Wachstum des etablierten Geschäfts erklärt. Dies ist jedoch wichtig, wenn man bedenkt, dass Einzelpersonen ihre Unternehmen aus einer Vielzahl von Gründen außer Wachstum oder Maximierung der wirtschaftlichen Rendite starten und betreiben (Wiklund et al. 2003).

Das Papier beginnt mit einer Analyse der Wachstumsabsichten des Unternehmens; Es folgt eine Bewertung des Einflusses der Geschlechtsidentität auf Wachstumsabsichten und die moderierende Rolle der Selbstkonstruktion. Als nächstes beschreiben wir unsere Stichprobe, Forschungsmethoden und Datenanalysetechniken, zu denen ANOVA und moderierte Mediationsanalysen gehören. Schließlich diskutieren wir die Ergebnisse der empirischen Analyse und die Grenzen der Forschung, präsentieren die praktischen Implikationen von Forschungsergebnissen und schlagen Bereiche für die weitere Forschung vor.

\section{Wachstumsabsicht und Geschlechtsidentität der Unternehmer}

Geschäftswachstum ist ein multidimensionales und komplexes Phänomen, das eine sorgfältige Planung und Einfängung seitens des Unternehmers erfordert. Als solches kann Geschäftswachstum als bewusstes und geplantes absichtliches Verhalten angesehen werden und folglich für Intentionsmodelle anwendbar sein (Ajzen, 1991; Krueger et al., 2000). Wachstumsabsichten sind von zentraler Bedeutung für das Verständnis des Geschäftswachstums, da sie der erste Schritt im Prozess sind und ein zielgerichtetes Element mit spezifischen Schritten zur Erreichung des Ziels beinhalten (Delmar \& Wiklund, 2008; Venugopal, 2016). Absichten wurden als Schlüsselprädiktor für tatsächliches Verhalten identifiziert, in einer Vielzahl von verschiedenen Verhaltensweisen, einschließlich Unternehmensgründung (Delmar \& Wiklund, 2008; Wiklund \& Shepherd, 2003). Darüber hinaus liefert die Forschung Hinweise darauf, dass der Anteil der Unternehmer mit Wachstumsabsichten an der Bevölkerung im Vergleich zu Selbstständigkeitsquoten oder allgemeinen Gründungsquoten ein signifikanter Prädiktor für das Wirtschaftswachstum ist (Stam et al., 2009).

Bis heute hat die Forschung über die Wachstumsabsichten von Unternehmern gemischte Ergebnisse in Bezug auf empirische Unterschiede zwischen Männern und Frauen hervorgebracht, und nicht alle Wissenschaftler sind sich einig, dass sich Frauen und Männer in ihrer allgemeinen Geschäftswachstumsabsicht unterscheiden (Cassar, 2006; Davis \& Shaver, 2012). Zum Beispiel fand Cassar (2006) heraus, dass Unternehmerinnen niedrigere Schätzungen der zukünftigen Einnahmen hatten als Männer. Menzies et al. (2004) fanden jedoch in einer in Kanada durchgeführten Studie keine statistisch signifikanten Unterschiede zwischen Frauen und Männern in ihren ausgedrückten Präferenzen für ein ungebremstes Wachstum. In der vorliegenden Studie schlagen wir vor, dass geschlechtsspezifische Sozialisationsprozesse Unterschiede zwischen den Wachstumsabsichten von männlichen und weiblichen Unternehmern erklären können.

Zeitgenössische Forschung im Unternehmertum legt nahe, dass Entscheidungen wie das Wachstum etablierter Unternehmen eng mit der Identität der Unternehmer verflochten sind (Shepherd \& Haynie 2009). Identität ist ein fließender sozialer Prozess, der eine Reihe von konzeptionellen Bedeutungen und theoretischen Rollen hat (Fauchart \& Gruber, 2011; Hoang \& Gimeno, 2010). Es kann als die Repräsentation der Verinnerlichung und Einbeziehung von sozial gehaltenen Verhaltenserwartungen durch die Menschen angesehen werden. Darüber hinaus kann Identität als grundlegendes Brückenkonzept zwischen individuum und sozial betrachtet werden (Hoang \& Gimeno, 2010). Individuen werden durch soziale Interaktionen sozial konstruiert und erwerben im Laufe ihres Lebens vielfältige und multiple soziale Identitäten. Im Falle des unternehmerischen Prozesses stellen Ireland und Webb (2007) in ihrer Rezension fest, dass der unternehmerische Prozess für Unternehmer auf Selbstidentitäten basiert und von ihnen angetrieben wird. Jüngste konzeptionelle Modelle der Rolle von Identität im Unternehmertum schlagen 
starke Verbindungen zwischen dem Selbstverständnis von Unternehmern und unternehmerischen Handlungen und Ergebnissen vor (Shepherd \& Haynie 2009), aber bis heute ist die empirische Forschung begrenzt (Farmer et al. 2011). Geschlechtsidentität gilt als wesentliches Element einer vielseitigen Selbstauffassung, die Individuen innerhalb sozialer Strukturen verortet (Eddleston \& Powell, 2008; Wood \& Eagly, 2010). Geschlecht unterscheidet sich von Geschlecht (Ahl, 2006). Forscher haben in den 1970er und 1980er Jahren Geschlecht von Geschlecht unterschieden, um zwischen biologischen Merkmalen von Männern und Frauen und den Bedeutungen zu unterscheiden, die Gesellschaften und Individuen männlichen und weiblichen Kategorien zuschreiben (Bem's Sex Role Inventory, 1981; Wood \& Eagly, 2010). Individuen werden während des gesamten Lebensverlaufs (implizit oder explizit) beigebracht, welche Verhaltensweisen und Rollen für Männer oder Frauen in der Gesellschaft wünschenswert sind. Daher akzeptieren oder verinnerlichen die meisten Menschen die kulturellen Bedeutungen, die mit ihren sexuellen Bedeutungen verbunden sind. Geschlechtsidentität stellt das Ausmaß dar, in dem eine Person glaubt, dass sie Merkmale besitzt, die mit traditionellen Geschlechterstereotypen verbunden sind (Bem's Sex Role Inventory, 1981; Wood \& Eagly, 2010).

Menschen unterscheiden sich darin, inwieweit sie Geschlechterrollen in ihr Selbstverständnis integrieren. Es entstehen zwei unabhängige Dimensionen der Geschlechtsidentität: Männlichkeit oder Überzeugungen darüber, inwieweit man Merkmale besitzt, die mit Männern verbunden sind (z. B. Dominanz, Unabhängigkeit, Ehrgeiz) und Weiblichkeit, oder Überzeugungen darüber, inwieweit man Merkmale besitzt, die mit Frauen verbunden sind (z. B. Sensibilität für die Bedürfnisse anderer, Mitgefühl, Verständnis) (Eddleston \& Powell, 2008; Wood \& Eagly, 2010). Frauen werden sozialisiert, um sich mit Attributen zu identifizieren, die als weiblich gelten, und Männer werden sozialisiert, um sich mit Attributen zu identifizieren, die als männlich gelten (Bem's Sex Role Inventory, 1981). Daher zeigen männliche Unternehmer eher eine stereotype männliche Orientierung und weibliche Unternehmer zeigen am ehesten eine stereotype weibliche Orientierung (Bird \& Brush, 2002).

Menschen verwenden ihre Geschlechtsidentität als Standard, um ihr Verhalten zu regulieren. Unternehmer, die ein männliches Selbstverständnis mit Unabhängigkeit und Dominanz haben, könnten ihr Verhalten regulieren, indem sie beispielsweise nach Möglichkeiten für hohes Wachstum und finanzielle Belohnungen aus ihren Unternehmen suchen (Bird \& Brush, 2002). Eddleston und Powell (2008) zeigen in ihrer Umfrage unter unternehmerischen Alumni, dass Unternehmerinnen andere Quellen der Beruflichen Zufriedenheit schätzen als Männer. Männliche Unternehmer bevorzugten Zufriedener, die mit der Statuserreichung verbunden sind (z. B. viel Geld verdienen, hohes Prestige und sozialen Status haben, in einer Führungsrolle sein, ein großes, schnell wachsendes Unternehmen führen), während Unternehmerinnen Zufriedenheitskräfte bevorzugten, die mit Mitarbeiterbeziehungen verbunden sind (z. B. mit freundlichen und sympathischen Menschen arbeiten, unterstützende Mitarbeiter haben, als Teil eines Teams arbeiten) und einen Beitrag zur Gesellschaft leisten. Dennoch legt die Studie keine Hinweise darauf vor, ob die Männlichkeit von Unternehmern stärker mit Wachstumsabsichten zusammenhängt als die Weiblichkeit von Unternehmern.

\section{Randbedingungen: Die Rolle Derunabhängigen Selbstkonstruktion}

Die Forschung liefert überzeugende Beweise dafür, dass das Konzept der unternehmerischen Tätigkeit geschlechtsspezifisch ist: Unternehmertum wird oft als eine Form von Männlichkeit dargestellt und die Begriffe "Unternehmer" und "männlich" sind tendenziell austauschbar geworden (Ahl \& Marlow 2012; Gupta et al., 2009). Dies unterstützt eine hierarchische Bewertung, bei der das Männliche gegenüber dem Weiblichen priorisiert wird und die Eigenschaften erfolgreicher Unternehmer (d.h. Proaktivität, Leistungsbedarf, Risikobereitschaft, Selbstvertrauen) stereotyp als männlich wahrgenommen werden (Ahl, 2006; Bird \& Brush, 2002; Coleman, 2016). Darüber hinaus wird das Verhalten von Frauen, die unternehmerisch tätig sind, nach den Standards einer unsichtbaren männlichen Norm definiert und bewertet (Lewis, 2006). Unternehmerinnen müssen gleichzeitig mit verschiedenen Identitäten umgehen, was zu Konflikten führen kann (Ahl, 2006; Bird \& Brush, 2002).

Dementsprechend müssen Frauen, um als glaubwürdige Akteure innerhalb des Unternehmertums anerkannt zu werden, das empfindliche Gleichgewicht zwischen der Annahme einer glaubwürdigen 
unternehmerischen Identität lernen, die maskulinisierte Normen widerspiegelt, ohne jedoch die zugeschriebene Weiblichkeit zu leugnen (Ahl \& Marlow 2012). Daher stellt sich eine wichtige Frage, wie die Wachstumsabsichten von Unternehmerinnen trotz der gemischten Botschaften, die sie von einem Prozess erhalten, der Männlichkeit breit betont, aber auch erwartet, dass Frauen sich speziell um Kinder kümmern und sie pflegen oder den Haushalt pflegen oder unterstützend sind, dh voneinander abhängig und mit anderen verbunden sind. Aufbauend auf den kulturellen Dimensionen von Individualismus und Kollektivismus schlagen Cross und Madson (1997) zusätzliche Facetten der Geschlechtsidentität vor, die Investitionen in ein individuelles versus soziales Selbstgefühl widerspiegeln. Die Autoren definieren männliche Identität als eigenständiges Selbstgefühl und weibliche Identität als interdependenten Selbstgefühl.

Insbesondere unabhängige und voneinander abhängige Selbstkonstruktionen stellen eines der grundlegendsten und übergreifendsten Schemata des Selbst eines Individuums dar (Markus \& Kitayama, 1991; Oyserman et al., 2002). Selbstkonstruktion bezieht sich auf die Art und Weise, wie ein Individuum sich selbst in Bezug auf andere Menschen versteht. Eine Person, die ein unabhängiges Selbstverständnis besitzt, betrachtet sich selbst als von anderen getrennt und zielt darauf ab, ein Gefühl der Autonomie und Einzigartigkeit zu bewahren. Eine Person, die eine voneinander abhängige Selbstkonstruktion besitzt, betrachtet sich selbst als mit anderen verbunden, woBei Selbstpräsentationen mit Darstellungen enger anderer und sozialer Kontexte verwoben werden (Markus \& Kitayama, 1991; Oyserman et al., 2002). Männer werden eher sozialisiert, um ein unabhängiges Selbstkonstrukt zu konstruieren und ein soziales Selbst zu entwickeln, das sich dadurch auszeichnen, dass sie den Wert auf Werte der individuellen Bedürfnisse, Autonomie und Selbstverwirklichung legen. Frauen werden eher sozialisiert, um ein voneinander abhängiges Selbstkonstrukt zu konstruieren und ein soziales Selbst zu entwickeln, das durch die Motivation gekennzeichnet ist, mit bestimmten Beziehungen oder Kontexten verbunden zu sein (Cross \& Madson, 1997; Oyserman et al., 2002; Singelis, 1994).

Es gibt bisher nur begrenzte Forschung, die die Beziehung zwischen Selbstkonstruktion und Unternehmertum bewertet hat. Jüngste empirische Forschungen liefern Beweise für die moderierende Rolle der Selbstkonstruktion auf individueller Ebene in kognitiven Modellen der Absicht, ein neues Unternehmen zu gründen (Siu \& Lo, 2013; Zampetakis et al., 2015). Zum Beispiel fanden Siu und Lo (2013) unter Verwendung einer Stichprobe von Studenten aus China und Hongkong heraus, dass die Stärke der wahrgenommenen sozialen Normen bei der Vorhersage unternehmerischer Absichten von einer voneinander abhängigen Selbstkonstruktion abhängt. Zampetakis et al. (2015) fanden unter Verwendung einer Stichprobe von Studenten aus Griechenland heraus, dass unabhängige Selbstkonstruktion mit Einstellungen zum Unternehmertum und moderierten Beziehungen zwischen Einstellungen und unternehmerischen Absichten zusammenhängt.

Wie bereits erwähnt, werden Frauen, um Legitimität als Unternehmerinnen zu erlangen, ermutigt, Einstellungen und Verhaltensweisen anzunehmen und $\mathrm{zu}$ reproduzieren, die in Wirklichkeit Reproduktionen dessen sind, was Männer tun und was Männer sind (Ahl \& Marlow, 2012). Einige Beweise deuten jedoch darauf hin, dass, wenn Frauen das Verhalten des idealisierten Mannes nachahmen, es Hinweise auf Misfit gibt. Zum Beispiel bewerteten Kerfoot und Miller (2010) die Ergebnisse eines Trainingsprogramms für potenzielle Unternehmer, das darauf abzielte, mehr Frauen zu ermutigen, ihre eigenen Unternehmungen zu gründen. Das Programm etablierte Männer als natürliche Unternehmer, die Frauen zu Außenseitern machten, und die einzige Hoffnung auf den Eintritt in den unternehmerischen Prozess bestand darin, zu lernen, wie man das Verhalten des idealisierten Mannes nachahmt. Nach dem Kurs wurden viele der potenziellen Unternehmerinnen aktiv davon abgehalten, neue Unternehmen zu gründen. Es zeigte sich, dass sie nicht zum maskulinisierten Bild eines Unternehmers "passten" (Kerfoot \& Miller, 2010).

Dies deutet darauf hin, dass, wenn Hinweise oder Symbole in der Umgebung unabhängige Selbstkonstruktion zugänglicher machen (Hong et al., 2000; Oyserman \& Sorensen, 2009) (d.h. Symbole für individuelle Freiheit, Autonomie, persönliche Erfüllung und Trennung), dann haben Unternehmerinnen aufgrund des Konflikts, der der Beziehung zwischen unabhängiger Selbstkonstruktion und Weiblichkeit innewohnt, möglicherweise weniger Bedarf an Wachstum ihrer Unternehmungen. Insbesondere Personen, 
die sich selbst als höher in der Weiblichkeit sehen, betrachten sich eher als Mitglied einer Gruppe. Dies wiederum führt $\mathrm{zu}$ einem kognitiven Stil, der sich mit den negativen Folgen von Verhalten (d. h. Geschäftswachstum) und der Vermeidung potenzieller Misserfolge befasst (Oyserman \& Sorensen, 2009). Daher erwarten wir, dass die Interaktion zwischen unabhängiger Selbstkonstruktion und Weiblichkeit für die Vorhersage von Wachstum negativ ist.

\section{Überblick überStudienziele und Hypothesen}

Ziel der vorliegenden Studie war es, Unterschiede im Prozess des Geschäftswachstums etablierter Unternehmen zwischen männlichen und weiblichen Unternehmern zu untersuchen. Wir konzentrierten uns auf einen bestimmten Aspekt des Unternehmenswachstums - die Absicht des Unternehmers - und ob und wie biologisches Geschlecht, Geschlechtsidentität und unabhängige Selbstkonstruktion die Wachstumsabsichten beeinflussen.

Konkret zielte die Studie zunächst darauf ab, festzustellen, ob und inwieweit das biologische Geschlecht mit der Geschlechtsidentität und den Wachstumsabsichten der Unternehmer zusammenhängt. Wir erwarteten, dass die Korrelation zwischen dem Geschlecht und der Geschlechtsidentität von Unternehmern so stark sein sollte, dass männliche Unternehmer am ehesten eine stereotype männliche Orientierung aufweisen und weibliche Unternehmer am ehesten eine stereotype weibliche Orientierung aufweisen. Zweitens wollten wir testen, ob und inwieweit Geschlechtsidentität biologische Geschlechtseffekte auf die Wachstumsabsichten von Unternehmen vermittelt. Wir erwarteten, dass geschlechtsidentität zumindest teilweise biologische Geschlechtseffekte auf Wachstumsabsichten vermitteln würde; Wir erwarteten, dass die Wirkung von Männlichkeit auf Wachstumsabsichten stärker sein würde als die Wirkung von Weiblichkeit. Schließlich untersuchten wir die moderierenden Einflüsse der unabhängigen Selbstkonstruktion, die Randbedingungen der Theorie darstellen. Wir erwarteten, dass eine unabhängige Selbstkonstruktion die Beziehung zwischen Geschlecht und Geschlechtsidentität sowie zwischen Geschlechtsidentität und Wachstumsabsichten moderieren würde. Unseres Wissens ist die vorliegende Studie eine der ersten, die kulturelle Orientierungen auf individueller Ebene innerhalb der Kultur als Vorläufer von Wachstumsabsichten eingehend untersucht.

Wir führten eine moderierte Mediationsanalyse durch (Hayes, 2013), um die mediatorische Kraft der Geschlechtsidentität und die moderierende Rolle der unabhängigen Selbstkonstruktion klar zu unterscheiden und gleichzeitig Variablen auf Unternehmensebene und auf individueller Ebene zu kontrollieren.

\section{SCHLUSSFOLGERUNG}

Die Ergebnisse der vorliegenden Studie machen deutlich, dass die Wachstumsabsicht ein komplexes Phänomen ist, das durch das Geschlecht beeinflusst werden kann. Unsere Ergebnisse zeigen, dass geschlechtsidentität den Einfluss von Sex auf Geschäftswachstumsabsichten vermittelt und die Mediationseffekte von der unabhängigen Selbstkonstruktion der Unternehmer abhängen. Daher treffen Frauen Entscheidungen im Zusammenhang mit dem Wachstum ihres Unternehmens mit einem anderen Prozess als Männer. Unsere Ergebnisse untermauern die Behauptung, dass es wertvoll ist, eine weibliche Perspektive zu berücksichtigen, wenn man die Faktoren untersucht, die die Wachstumsabsichten von Unternehmern im etablierten Geschäft beeinflussen. 


\section{TRANSLATED VERSION: PORTUGUESE}

Below is a rough translation of the insights presented above. This was done to give a general understanding of the ideas presented in the paper. Please excuse any grammatical mistakes and do not hold the original authors responsible for these mistakes.

\section{VERSÃO TRADUZIDA: PORTUGUÊS}

Aqui está uma tradução aproximada das ideias acima apresentadas. Isto foi feito para dar uma compreensão geral das ideias apresentadas no documento. Por favor, desculpe todos os erros gramaticais e não responsacule os autores originais responsáveis por estes erros.

\section{INTRODUÇÃO}

O desenvolvimento e o crescimento dos negócios existentes é um processo com muitos benefícios sociais, incluindo a criação de emprego e riqueza e o avanço da inovação (Tang \& Koveos, 2004; Van Praag \& Versloot, 2007). A decisão dos empreendedores de crescer seus negócios é complexa, não é linear nem depende de um número limitado de fatores (Miller et al. 2013). Os empreendedores não só têm que tomar decisões importantes para diferentes tarefas que são de natureza diferente, mas também têm que garantir que essas decisões sejam as certas não apenas para seus negócios, mas também para si mesmos. Enquanto alguns empreendedores têm o prazer de serem autônomos em pequena escala, outros têm aspirações de crescimento para seus empreendimentos (Shane, 2009).

Pesquisas claramente delineiam que as empresas de propriedade feminina tendem a ter níveis mais baixos de crescimento e permanecem menores do que as empresas de propriedade masculina (Cliff, 1998; Coleman, 2016; Davis \& Shaver, 2012), sugerindo a existência de uma lacuna entre homens e mulheres no empreendedorismo; esta é uma questão que está atraindo cada vez mais atenção acadêmica (Coleman, 2016). No entanto, grande parte dessa pesquisa investigou diferenças nos níveis de atividade inicial entre homens e mulheres (ver Jennings \& Brush, 2013). Pouca pesquisa tem examinado as aspirações de crescimento dos empreendimentos liderados por mulheres. Em comparação com os empreendedores do sexo masculino, as empreendedoras tendem a perseguir metas não econômicas, como equilibrar o trabalho e os papéis familiares e têm preferências pelo relacionamento dos funcionários e satisfações da sociedade que, por sua vez, podem prejudicar o desempenho econômico ou o crescimento (Eddleston \& Powell, 2008; Jennings \& Brush, 2013).

Como observou Henry et al. (2016), as representações normativas do empreendedorismo são dominadas pela masculinidade. Isso marginaliza as empreendedoras e as torna invisíveis. Os autores observam que, embora a consciência de que o gênero (masculino e feminino) seja socialmente construído e corresponda a um conjunto aprendido de comportamentos que não se referem simplesmente ao sexo biológico (homem/homem e mulher/mulher) do empreendedor parece difundido entre os estudiosos, vários temas prejudiciais relacionados às mulheres são persistentes. Por exemplo, muitos estudiosos usam normas masculinas para julgar as atividades das mulheres meramente comparando homens e mulheres, com pouca ou nenhuma atenção prestada às construções de gênero (Ahl, 2006; Lewis, 2006). No entanto, essa prática negligencia o fato de que aspectos masculinos e femininos serão incorporados ao processo de crescimento dos negócios para homens e mulheres. Ou seja, o gênero pode ser um aspecto vital da intenção de crescimento dos negócios.

Juntas, até o momento, a pesquisa não fornece explicações conclusivas para a variação da trajetória de crescimento que homens e mulheres gostariam que seu empreendimento seguisse (ou seja, sua intenção de crescimento) (Bulanova et al. 2016; Dutta \& Thornhill, 2008). A intenção de crescimento é considerada uma característica essencial do comportamento empreendedor e um elemento-chave na compreensão do desenvolvimento e crescimento do empreendimento (Sadler-Smith et al. 2003). Além disso, as intenções provaram ser o melhor preditor do comportamento planejado (Ajzen, 1991), especialmente se o fenômeno envolve atrasos de tempo imprevisíveis, planejamento e alto grau de processamento cognitivo, como o 
crescimento dos negócios (Krueger et al. 2000). As intenções de crescimento são consideradas um preditorchave do crescimento real dos negócios (Delmar \& Wiklund, 2008; Wiklund \& Shepherd, 2003).

Evidências consideráveis sugerem que a identidade de um indivíduo (ou conceito de si mesmo) em geral (Fauchart \& Gruber, 2011; Hoang \& Gimeno, 2010) e identidade de gênero (ou papéis de gênero relacionados) em particular (Eddleston \& Powell, 2008; Gupta et al., 2009) podem contribuir para diferenças substanciais entre homens e mulheres no comportamento empreendedor. As pessoas tendem a diferir na medida em que incorporam papéis de gênero em seus autoconceitos.

No presente estudo, propomos que a identidade de gênero dos empreendedores ou até que ponto os empreendedores possuam traços associados aos estereótipos tradicionais de gênero (Inventário de Papel Sexual do Bem, 1981; Mueller, 2004; Wood \& Eagly, 2010) é um importante mecanismo cognitivo que relaciona o sexo dos empreendedores às intenções de crescimento dos negócios. Argumentamos que a identidade de gênero molda a forma como os empreendedores se veem, como eles entendem o mundo ao seu redor e se aproximam de outras pessoas, mas também o que eles pretendem alcançar no futuro (Eddleston \& Powell, 2008; Hoang \& Gimeno, 2010).

No entanto, os mecanismos pelos quais a identidade sexual e de gênero exercem influência sobre as intenções de crescimento dos empreendedores e as influências moderadoras que constituem condições de fronteira da teoria precisam de uma investigação mais aprofundada. A auto-construção é uma moderadora potencial da relação entre gênero, identidade de gênero e intenções de crescimento. Auto-construtivo referese aos pensamentos, sentimentos e ações culturalmente contingentes dos indivíduos que se preocupam com a compreensão do eu como conectado aos outros (auto-construtivo interdependente) ou distintos dos outros (auto-construtivo independente) (Markus \& Kitayama, 1991). A exposição dos indivíduos a determinadas condições pode aumentar a probabilidade de apresentarem tendências independentes (individualistas) ou interdependentes (coletivistas) (Hong et al., 2000). Os conceitos de "empreendedorismo" e "empreendedorismo" são considerados de gênero masculino (Ahl, 2006; Lewis, 2006). Pistas ou símbolos no ambiente que os empreendedores utilizam para operar seus negócios poderiam tornar a auto-construção independente mais acessível (Hong et al., 2000), já que a identidade masculina destaca atributos únicos dos indivíduos (Cross \& Madson, 1997). Uma consideração sistemática dos potenciais efeitos moderados da auto-construção contribuirá para uma compreensão mais completa das condições sob as quais os efeitos do sexo sobre as intenções de crescimento são mais propensos a manter.

Em suma, o objetivo deste artigo é aprofundar a compreensão dos fatores que influenciam a motivação do crescimento dos negócios em empresas estabelecidas. Para isso, um dos principais objetivos do presente estudo é examinar a relação entre sexo dos empreendedores, identidade de gênero e as intenções de crescimento dos empresários de seus empreendimentos estabelecidos. Argumentamos que uma exploração nas intenções de crescimento dos empresários de empresas estabelecidas poderia ajudar a explicar as diferenças sexuais na decisão de crescer. Um segundo grande objetivo do presente estudo é focar na autointerpretação dos empreendedores como um potencial moderador da relação entre sexo e identidade de gênero e entre identidade de gênero e intenção de crescimento empresarial (ver modelo conceitual na Fig. 1). Até onde sabemos, até o momento, tem havido pouca pesquisa explicando a influência desses domínios e a intenção dos empreendedores para o crescimento do negócio estabelecido. No entanto, isso é importante considerando que os indivíduos iniciam e operam seus empreendimentos por uma variedade de razões além do crescimento ou maximização dos retornos econômicos (Wiklund et al. 2003).

O artigo começa com uma análise das intenções de crescimento dos negócios; isso é seguido por uma avaliação da influência da identidade de gênero nas intenções de crescimento e do papel moderador da autoconstrução. Em seguida, descrevemos nossas técnicas de amostra, métodos de pesquisa e análise de dados que incluem análises de mediação ANOVA e moderadas. Por fim, discutimos os resultados da análise empírica e as limitações da pesquisa, apresentamos a implicação prática dos resultados da pesquisa e propomos áreas para novas pesquisas.

\section{Intenção de Crescimentoe Identidade de Gênero dos Empreendedores}

$\mathrm{O}$ crescimento do negócio é um fenômeno multidimensional e complexo que envolve planejamento cuidadoso e pensamento por parte do empreendedor. Como tal, o crescimento dos negócios pode ser 
considerado um comportamento intencional deliberado e planejado e, consequentemente, aplicável para modelos de intenção (Ajzen, 1991; Krueger et al., 2000). As intenções de crescimento são fundamentais para a compreensão do crescimento dos negócios, pois são o primeiro passo do processo e envolvem um elemento purposivo com etapas específicas para atingir a meta (Delmar \& Wiklund, 2008; Venugopal, 2016). As intenções foram identificadas como um preditor-chave do comportamento real, em uma ampla gama de comportamentos diferentes, incluindo a startup de negócios (Delmar \& Wiklund, 2008; Wiklund $\&$ Shepherd, 2003). Além disso, a pesquisa fornece evidências de que a proporção de empreendedores com intenções de crescimento na população é um preditor significativo de crescimento econômico em comparação com as taxas de auto-emprego ou taxas gerais de start-up (Stam et al., 2009).

Até o momento, pesquisas sobre as intenções de crescimento dos empreendedores produziram resultados mistos sobre diferenças empíricas entre homens e mulheres e nem todos os estudiosos concordam que mulheres e homens diferem em sua intenção global de crescimento dos negócios (Cassar, 2006; Davis \& Shaver, 2012). Por exemplo, Cassar (2006) descobriu que as mulheres empreendedoras tinham estimativas mais baixas de receitas futuras do que os homens. No entanto, Menzies et al. (2004), em um estudo realizado no Canadá, não encontraram diferenças estatisticamente significativas entre mulheres e homens em suas preferências expressas de crescimento desenfreado. No presente estudo, propomos que os processos de socialização de gênero possam explicar diferenças entre as intenções de crescimento empresarial dos empresários homens e mulheres.

Pesquisas contemporâneas em empreendedorismo sugerem que decisões como o crescimento de empreendimentos estabelecidos estão intimamente entrelaçadas com a identidade dos empreendedores (Shepherd \& Haynie 2009). A identidade é um processo social fluido que tem uma gama de significados conceituais e funções teóricas associadas a ela (Fauchart \& Gruber, 2011; Hoang \& Gimeno, 2010). Pode ser vista como representação dos povos da internalização e incorporação de expectativas comportamentais socialmente mantidas. Além disso, a identidade pode ser considerada como um conceito fundamental de ponte entre o indivíduo e o social (Hoang \& Gimeno, 2010). Os indivíduos são socialmente construídos através de interações sociais e que adquirem ao longo de suas vidas diversas e múltiplas identidades sociais. No caso do processo empreendedor, Irlanda e Webb (2007) observam em sua revisão que, para os empreendedores, o processo empreendedor é baseado e impulsionado por auto-identidades. Modelos conceituais recentes do papel da identidade no empreendedorismo propõem fortes laços entre o autoconceito dos empreendedores e ações e resultados empreendedores (Shepherd \& Haynie 2009), mas até o momento, a pesquisa empírica é limitada (Farmer et al. 2011). A identidade de gênero é considerada um elemento essencial de uma concepção de muitos lados do eu que situa os indivíduos dentro das estruturas sociais (Eddleston \& Powell, 2008; Wood \& Eagly, 2010). Gênero é diferente do sexo (Ahl, 2006). Pesquisadores distinguiram o sexo do gênero durante as décadas de 1970 e 1980 para distinguir entre características biológicas de homens e mulheres e os significados que sociedades e indivíduos atribuem às categorias masculina e feminina (Inventário de Papel Sexual de Bem, 1981; Wood \& Eagly, 2010). Os indivíduos ao longo do curso da vida são ensinados (implicitamente ou explicitamente) quais comportamentos e papéis são desejáveis para homens ou mulheres na sociedade. Como tal, a maioria dos indivíduos aceita ou internaliza os significados culturais associados aos seus significados sexuais. A identidade de gênero representa até que ponto um indivíduo acredita que possui traços associados aos estereótipos tradicionais de gênero (Inventário de Papel Sexual de Bem, 1981; Wood \& Eagly, 2010).

As pessoas diferem na medida em que incorporam papéis de gênero em seus autoconceitos. Duas dimensões independentes da identidade de gênero surgem: masculinidade, ou crenças sobre até que ponto se possui traços associados aos homens (por exemplo, dominância, independência, ambição) e feminilidade, ou crenças sobre até que ponto se possui traços associados às fêmeas (por exemplo, sensibilidade às necessidades dos outros, compaixão, compreensão) (Eddleston \& Powell, 2008; Wood \& Eagly, 2010). As mulheres são socializadas para se identificar com atributos considerados femininos, e os homens são socializados para se identificar com atributos considerados masculinos (Inventário de Papel Sexual de Bem, 1981). Como tal, empreendedores masculinos são mais propensos a exibir uma orientação masculina estereotipada e empreendedoras femininas são mais propensas a exibir uma orientação feminina estereotipada (Bird \& Brush, 2002). 
As pessoas usam sua identidade de gênero como um padrão contra o qual regular seu comportamento. Empreendedores que têm um autoconceito masculino envolvendo independência e dominância podem regular seu comportamento, por exemplo, buscando oportunidades de alto crescimento e recompensas financeiras de seus negócios (Bird \& Brush, 2002). Eddleston e Powell (2008) em sua pesquisa de exalunos empreendedores mostram que as mulheres empreendedoras valorizam diferentes fontes de satisfação na carreira do que os homens. Os empreendedores do sexo masculino preferiram satisfações associadas à obtenção de status (por exemplo, ganhar muito dinheiro, ter alto prestígio e status social, estar em um papel de liderança, liderar uma grande empresa em rápido crescimento) enquanto as empreendedoras preferem satisfações associadas às relações com os funcionários (por exemplo, trabalhar com pessoas amigáveis e agradáveis, ter funcionários solidários, trabalhar como parte de uma equipe) e contribuir para a sociedade. No entanto, o estudo não apresenta evidências sobre se a masculinidade dos empresários se relaciona mais fortemente com as intenções de crescimento em comparação com a feminilidade dos empresários.

\section{Condições de fronteira: $O$ papel of Independente Auto-Construtivo}

A pesquisa fornece evidências convincentes de que o conceito de atividade empreendedora é tendencioso de gênero: $O$ empreendedorismo é frequentemente retratado como uma forma de masculinidade e os termos "empreendedor" e "masculino" tendem a se tornar intercambiáveis (Ahl \& Marlow 2012; Gupta et al., 2009). Isso sustenta uma valorização hierárquica na qual o masculino é priorizado sobre o feminino e as características dos donos de negócios bem-sucedidos (ou seja, proatividade, necessidade de realização, risco de competir, confiança) são estereotipadas percebidas como masculinas (Ahl, 2006; Bird \& Brush, 2002; Coleman, 2016). Além disso, o comportamento das mulheres envolvidas na atividade empreendedora é definido e avaliado de acordo com os padrões de uma norma masculina invisível (Lewis, 2006). Mulheres empreendedoras têm que gerenciar diferentes identidades simultaneamente que podem resultar em conflitos (Ahl, 2006; Bird \& Brush, 2002).

Assim, para serem reconhecidas como atores críveis dentro do empreendedorismo, as mulheres têm que aprender o delicado equilíbrio entre adotar uma identidade empreendedora crível que reflete normas masculinizadas, mas sem negar a feminilidade atribuída (Ahl \& Marlow 2012). Assim, uma questão importante levantada é como as intenções de crescimento das mulheres empreendedoras são formadas apesar das mensagens mistas que recebem de um processo que enfatiza amplamente a masculinidade, mas também espera que as mulheres cuidem e alimentem e alimentem as crianças ou mantenham o lar, ou sejam solidárias, ou seja, interdependentes e conectadas com outras pessoas. A partir das dimensões culturais do individualismo e do coletivismo, Cross e Madson (1997) propõem facetas adicionais da identidade de gênero refletindo o investimento em um sentido individual versus social de si mesmo. Os autores definem a identidade masculina como um senso independente de auto e identidade feminina como um sentido interdependente de si mesmo.

Especificamente, auto-construcionais independentes e interdependentes representam uma das mais fundamentais e abrangentes esquemas da autoestima de um indivíduo (Markus \& Kitayama, 1991; Oyserman et al., 2002). Auto-construtivo refere-se à forma como um indivíduo se entende em relação a outras pessoas. Uma pessoa que possui uma auto-construção independente vê-se separada das outras, com o objetivo de manter um senso de autonomia e singularidade. Uma pessoa que possui uma autointerpretação interdependente vê-se como conectado aos outros, onde as auto-apresentações são tecidas juntamente com representações de outros próximos e contexto social (Markus \& Kitayama, 1991; Oyserman et al., 2002). Os homens são mais propensos a serem socializados para construir uma autoconstrução independente e desenvolver um eu social que seja marcado por dar ênfase primária aos valores das necessidades individuais, autonomia e auto-realização. As mulheres são mais propensas a serem socializadas para construir uma auto-construção interdependente e desenvolver um eu social que seja marcado pela motivação de estar conectada com relações ou contextos particulares (Cross \& Madson, 1997; Oyserman et al., 2002; Singelis, 1994).

Há pesquisas limitadas até o momento que avaliaram a relação entre auto-construtivo e empreendedorismo. Pesquisas empíricas recentes fornecem evidências para o papel moderador da autoconstrução individual em modelos cognitivos de intenção de iniciar um novo negócio (Siu \& Lo, 2013; 
Zampetakis et al., 2015). Por exemplo, Siu e Lo (2013), usando uma amostra de estudantes da China e hong kong, descobriram que a força das normas sociais percebidas na previsão da intenção empreendedora dependia da auto-construção interdependente. Zampetakis et al. (2015) utilizando uma amostra de estudantes da Grécia descobriram que a auto-construção independente estava relacionada a atitudes em relação ao empreendedorismo e relações moderadas entre atitudes e intenções empreendedoras.

Como observado acima, para que as mulheres ganhem legitimidade como empreendedoras, elas são encorajadas a adotar e reproduzir atitudes e comportamentos que são, de fato, reproduções do que os homens fazem e do que os homens são (Ahl \& Marlow, 2012). No entanto, algumas evidências sugerem que quando as mulheres imitam o comportamento do homem idealizado, há evidências de desajustado. Por exemplo, Kerfoot e Miller (2010) avaliaram os resultados de um programa de treinamento para potenciais donos de negócios com o objetivo de incentivar mais mulheres a iniciar seus próprios empreendimentos. $\mathrm{O}$ programa estabeleceu homens como empreendedores naturais tornando as mulheres como forasteiras, e a única esperança de entrada no processo empreendedor foi aprendendo a emular o comportamento do homem idealizado. Após a realização do curso, muitas das potenciais proprietárias de negócios foram ativamente desencorajadas a iniciar novos empreendimentos. Ficou evidente que eles não "se encaixavam" na imagem masculinizada de um empreendedor (Kerfoot \& Miller, 2010).

Isso sugere que quando as pistas ou símbolos no ambiente tornam a auto-construção independente mais acessível (Hong et al., 2000; Oyserman \& Sorensen, 2009) (ou seja, símbolos de liberdade individual, autonomia, realização pessoal e separação), então os empreendedores femininos podem ter menos necessidade de crescer seus empreendimentos por causa do conflito inerente à relação entre autoconstrutivo independente e feminilidade. Especificamente, indivíduos que se veem como mais elevados na feminilidade são mais propensos a se considerarem como um membro de um grupo. Isso, por sua vez, provoca um estilo cognitivo que se preocupa com as consequências negativas do comportamento (que é o crescimento dos negócios) e evitando um possível fracasso (Oyserman \& Sorensen, 2009). Assim, esperamos que a interação entre autodestrutivo independente e feminilidade para a previsão de crescimento seja negativa.

\section{Visão geral of Estudo Visa umae hipóteses}

O objetivo do presente estudo foi examinar diferenças no processo de crescimento dos negócios de empresas estabelecidas, entre empresários do sexo masculino e feminino. Focamos em um aspecto específico do crescimento firme - a intenção do empreendedor - e se e como sexo biológico, identidade de gênero e auto-construtivo independente afetam as intenções de crescimento.

Especificamente, o estudo teve como objetivo determinar, em primeiro lugar, se e até que ponto o sexo biológico está associado à identidade de gênero e às intenções de crescimento empresarial dos empreendedores. Esperávamos que a correlação entre o sexo dos empreendedores e a identidade de gênero fosse forte, de modo que os empreendedores masculinos são mais propensos a exibir uma orientação masculina estereotipada e as empreendedoras femininas são mais propensas a exibir uma orientação feminina estereotipada. Em segundo lugar, pretendemos testar se, e até que ponto, a identidade de gênero media os efeitos sexuais biológicos nas intenções de crescimento dos negócios. Esperávamos que a identidade de gênero mediaria pelo menos parcialmente os efeitos sexuais biológicos nas intenções de crescimento; esperávamos que o efeito da masculinidade nas intenções de crescimento fosse mais forte em comparação com o efeito da feminilidade. Finalmente, examinamos as influências moderadoras da autoconstrução independente que constituem condições de fronteira da teoria. Esperávamos que a autointerpretação independente moderasse a relação entre sexo e identidade de gênero e entre identidade de gênero e intenções de crescimento. Para nosso conhecimento, o presente estudo é um dos primeiros a examinar as orientações culturais individuais dentro da cultura como antecedentes às intenções de crescimento empresarial em profundidade.

Realizamos uma análise moderada de mediação (Hayes, 2013) a fim de distinguir claramente o poder mediador da identidade de gênero e o papel moderador da auto-construção independente enquanto controla para variáveis de nível firme e individual. 


\section{CONCLUSÃO}

Os achados do presente estudo deixam claro que a intenção de crescimento empresarial é um fenômeno complexo que pode ser influenciado pelo gênero. Nossos resultados indicam que a identidade de gênero media a influência do sexo nas intenções de crescimento dos negócios e os efeitos da mediação dependem da auto-construção independente dos empreendedores. Assim, as mulheres tomam decisões relacionadas ao crescimento de seus negócios usando um processo diferente dos homens. Nossos resultados reforçam as afirmações de que é valioso incorporar uma perspectiva feminina ao estudar os fatores que influenciam as intenções de crescimento dos empreendedores nos negócios estabelecidos. 